\title{
Dipole operator constraints on composite Higgs models
}

\author{
Matthias König ${ }^{1, a}$, Matthias Neubert ${ }^{1,2, b}$, David M. Straub ${ }^{3, c}$ \\ ${ }^{1}$ PRISMA Cluster of Excellence and Mainz Institute for Theoretical Physics, Johannes Gutenberg University, 55099 Mainz, Germany \\ 2 Department of Physics, LEPP, Cornell University, Ithaca, NY 14853, USA \\ ${ }^{3}$ Excellence Cluster Universe, Technische Universität München, Boltzmannstr. 2, 85748 Garching, Germany
}

Received: 25 March 2014 / Accepted: 17 June 2014 / Published online: 23 July 2014

(C) The Author(s) 2014. This article is published with open access at Springerlink.com

\begin{abstract}
Flavour- and CP-violating electromagnetic or chromomagnetic dipole operators in the quark sector are generated in a large class of new physics models and are strongly constrained by measurements of the neutron electric dipole moment and observables sensitive to flavour-changing neutral currents, such as the $B \rightarrow X_{s} \gamma$ branching ratio and $\epsilon^{\prime} / \epsilon$. After a model-independent discussion of the relevant constraints, we analyze these effects in models with partial compositeness, where the quarks get their masses by mixing with vector-like composite fermions. These scenarios can be seen as the low-energy limit of composite Higgs or warped extra dimensional models. We study different choices for the electroweak representations of the composite fermions motivated by electroweak precision tests as well as different flavour structures, including flavour anarchy and $U(3)^{3}$ or $U(2)^{3}$ flavour symmetries in the strong sector. In models with "wrong-chirality" Yukawa couplings, we find a strong bound from the neutron electric dipole moment, irrespective of the flavour structure. In the case of flavour anarchy, we also find strong bounds from flavour-violating dipoles, while these constraints are mild in the flavour-symmetric models.
\end{abstract}

\section{Introduction}

The discovery of the Higgs boson [1,2] has made the question what stabilises the electroweak scale more acute and has reduced the implementation of weak-scale naturalness to two possibilities: supersymmetry and Higgs compositeness. In this paper, we study the latter possibility, which arguably has received less attention in the literature. This is in part due to the difficulty in treating the strong interactions that are responsible for the Higgs bound state. Much progress has been made in recent years by warped compactifications of

\footnotetext{
a e-mail: koenigma@thep.physik.uni-mainz.de

be-mail: neubertm@uni-mainz.de

c e-mail: david.straub@tum.de
}

higher-dimensional space-times [3-5], providing a weakly coupled dual description of the strong interactions in four dimensions. On the other hand, purely four-dimensional (4D) models have been constructed as well [6-10], with a particularly well-motivated example being models in which the Higgs arises as a pseudo Nambu-Goldstone boson, explaining its lightness with respect to the other, as yet unobserved, resonances. ${ }^{1}$

In all these models, indirect constraints from low-energy precision observables play a crucial role. Generating fermion masses without excessive flavour violation singles out the mechanism of partial compositeness where, from a 4D effective theory point of view, the elementary Standard Model (SM) fermions obtain masses by mixing linearly with composite vector-like fermion resonances [12]. Since the degree of compositeness is required to be smaller for light quarks, tree-level flavour-changing neutral currents (FCNCs) mediated by composite resonances are automatically suppressed [13-15]. However, unless one is willing to accept a fine tuning of a few per cent, for $\mathrm{TeV}$-scale resonances this suppression mechanism is not quite strong enough to suppress $\mathrm{CP}$ violation in kaon mixing, if no additional flavour symmetry is assumed [16-19]. In addition to flavour constraints, the mixing of the SM fermions with composite states with different electroweak quantum numbers leads to potentially large corrections to electroweak precision observables. In particular, custodial symmetry should be imposed on the strong sector to avoid a tree-level correction to the $T$ parameter, and the representations of the composite fermions have to be chosen to avoid large tree-level corrections to the $Z \bar{b}_{L} b_{L}$ vertex [20,21].

In addition to electroweak precision observables and treelevel flavour-changing processes, an important class of constraints on these models is given by loop-induced dipole operators that contribute to radiative FCNC decays or fermionic

\footnotetext{
$\overline{1}$ See also [11] for a recent review of the composite Higgs model landscape in the light of the Higgs discovery.
} 
dipole moments. The presence of heavy vector-like fermions charged under the electroweak gauge group implies a potential enhancement of these chirality-violating operators compared to the SM. A number of studies of dipole operators have been presented in the literature, either for RandallSundrum models in the KK basis [16,22-25], for purely 4D models [26], or genuine five-dimensional (5D) calculations [27-29]. The aim of this work is to exploit the computational simplicity of the 4D models to study the impact of different choices for the fermion representations and of different flavour symmetries on the constraints from observables sensitive to dipole operators. To this end, we will use a generalisation of the framework of Ref. [6], considering one set of composite partners for each SM field, plus the additional states required for custodial protection of $T$ and $Z \rightarrow b_{L} \bar{b}_{L}$. Our work can be seen as a complement to similar studies of electroweak and tree-level flavour constraints [30] and Zmediated rare decays [31] in the same models.

The remainder of this paper is organised as follows. In Sect. 2 we define our model setup. Section 3 is devoted to a model-independent discussion of dipole operators, the observables probing them and the constraints obtained from existing measurements. In Sect. 4 we derive approximate analytical expressions for the leading contributions to the Wilson coefficients of the dipole operators within our setup and estimate the size of subleading contributions. These results are then used in Sect. 5 to obtain numerical bounds on the masses of composite resonances derived from the various observables sensitive to dipole operators. While depending weakly on the choice of fermion representations, these bounds will depend strongly on the presence or not of a flavour symmetry in the strong sector. We will discuss the explicit examples of a $U(3)^{3}$ or a $U(2)^{3}$ flavour symmetry, only broken by the left- or right-handed composite-elementary mixings [32-35]. Since some results in the literature partially overlap with our results, it is mandatory that we compare our findings to them; we do this in Sect. 6. Section 7 contains our conclusions.

\section{Setup}

We consider simple 4D models, in which partial compositeness is implemented along the lines of Ref. $[6]^{2}$ : the SM field content (without the Higgs) is complemented by a composite Higgs, a set of vector resonances transforming under the global symmetry $G_{c}=S U(3)_{c} \times S U(2)_{L} \times S U(2)_{R} \times$ $U(1)_{X}$ and a set of fermion resonances that fill complete representations of $G_{c}$. SM fermions and gauge bosons obtain

\footnotetext{
${ }^{2}$ See Sect. 7 for a comment on the applicability of our discussion of more fundamental models, where the Higgs arises as a pseudo NambuGoldstone boson.
}

masses from linear mixing terms with the composite states. Since $G_{c}$ is larger than the SM gauge group, there is some freedom in the choice of the fermion representations. In addition to the simplest case, involving just one $S U(2)_{L}$ doublet and one $S U(2)_{R}$ doublet ("doublet model"), we consider two cases ("triplet model", "bidoublet model"), which are motivated by the custodial protection of the $Z b \bar{b}$ coupling.

Using a notation where lower-case letters refer to elementary fields, while upper-case letters denote composite states, the part of the Lagrangian involving fermions reads

- In the doublet model,

$$
\begin{aligned}
& \mathcal{L}_{s}^{\text {doublet }}=-\bar{Q}_{a}^{i} m_{Q}^{i} Q_{a}^{i}-\bar{R}_{a}^{i} m_{R}^{i} R_{a}^{i} \\
& -\left(Y^{i j} \bar{Q}_{L a}^{i} \mathcal{H}_{a b} R_{R b}^{j}+\tilde{Y}^{i j} \bar{R}_{L a}^{i} \mathcal{H}_{b a}^{*} Q_{R b}^{j}+\text { h.c. }\right), \\
& \mathcal{L}_{\text {mix }}^{\text {doublet }}=\lambda_{L}^{i j} \bar{q}_{L a}^{i} Q_{R a}^{j}+\lambda_{R u}^{i j} \bar{U}_{L}^{i} t_{R}^{j}+\lambda_{R d}^{i j} \bar{D}_{L}^{i} b_{R}^{j},
\end{aligned}
$$

where $\mathcal{H}=\left(i \sigma_{2} H^{*}, H\right)$ is the Higgs bidoublet, $Q=$ $\left(\begin{array}{ll}T & B\end{array}\right)^{\mathrm{T}}$ transforms as a $(\mathbf{2}, \mathbf{1})_{1 / 6}$ under $S U(2)_{L} \times$ $S U(2)_{R} \times U(1)_{X}$, and $R=(U D)$ transforms as a $(\mathbf{1}, \mathbf{2})_{1 / 6}$. Here and in the following, $i, j$ are flavour indices and $a, b, c$ are $S U(2)_{L}$ or $S U(2)_{R}$ indices. Fermion fields without a chirality index are to be understood as $\psi=$ $\psi_{L}+\psi_{R}$, so that the mass terms for these fields are of the form $\bar{\psi} m_{\psi} \psi=m_{\psi}\left(\bar{\psi}_{L} \psi_{R}+\bar{\psi}_{R} \psi_{L}\right)$.

- In the triplet model,

$$
\begin{aligned}
& \mathcal{L}_{s}^{\text {triplet }}=\bar{L}_{a b}^{i} m_{Q_{u}}^{i} L_{b a}^{i}-\bar{R}_{a}^{i} m_{R}^{i} R_{a}^{i}-\bar{R}_{a}^{\prime i} m_{R}^{i} R_{a}^{\prime i} \\
& \quad-\left[Y^{i j}\left(\bar{L}_{L}^{i}\right)_{a b} \mathcal{H}_{b c}\left(R_{R}^{j}\right)_{c a}+Y^{i j}\left(\bar{L}_{L}^{i}\right)_{a b} \mathcal{H}_{b c}\left(R_{R}^{\prime j}\right)_{c a}\right. \\
& \left.\quad+\tilde{Y}^{i j}\left(\bar{R}_{L}^{i}\right)_{a b} \mathcal{H}_{c b}^{*}\left(L_{R}^{j}\right)_{c a}+\tilde{Y}^{i j}\left(\bar{R}_{L}^{i}\right)_{a b} \mathcal{H}_{c b}^{*}\left(L_{R}^{j}\right)_{c a}+\text { h.c. }\right], \\
& \mathcal{L}_{\text {mix }}^{\text {triplet }}=\lambda_{L}^{i j} \bar{q}_{L a}^{i} Q_{R a}^{j}+\lambda_{R u}^{i j} \bar{U}_{L}^{i} t_{R}^{j}+\lambda_{R d}^{i j} \bar{D}_{L}^{i} b_{R}^{j},
\end{aligned}
$$

where $L$ is a bidoublet transforming as a $(\mathbf{2}, \mathbf{2})_{2 / 3}$, and $R$ and $R^{\prime}$ are $S U(2)_{L}$ and $S U(2)_{R}$ triplets, transforming as $(\mathbf{3}, \mathbf{1})_{2 / 3}$ and $(\mathbf{1}, \mathbf{3})_{2 / 3}$, respectively. In component notation, the multiplets are given by

$$
\begin{gathered}
L=\left(Q Q^{\prime}\right)=\left(\begin{array}{ll}
T & T_{5 / 3} \\
B & T_{2 / 3}
\end{array}\right), \quad R=\left(\begin{array}{lll}
U_{5 / 3} & U & D
\end{array}\right)^{\mathrm{T}}, \\
R^{\prime}=\left(\begin{array}{l}
U_{5 / 3}^{\prime} U^{\prime} D^{\prime}
\end{array}\right) .
\end{gathered}
$$

In the Yukawa couplings, we have also used the triplets rewritten as $2 \times 2$ matrices, $R_{a b}^{(\prime)}=\tau_{a b}^{\alpha} R_{\alpha}^{(\prime)}$ with $\tau^{1,2}=$ $\left(\sigma^{1} \pm i \sigma^{2}\right) / 2$ and $\tau^{3}=\sigma^{3} / \sqrt{2}$.

- In the bidoublet model

$$
\mathcal{L}_{s}^{\text {bidoublet }}=-\left(\bar{L}_{U}^{i}\right)_{a b}^{i} m_{Q_{u}}^{i}\left(L_{U}^{i}\right)_{b a}-\bar{U}^{i} m_{U}^{i} U^{i}
$$




$$
\begin{aligned}
& +\left[Y_{U}^{i j}\left(\bar{L}_{U, L}^{i}\right)_{a b} \mathcal{H}_{b a} U_{R}^{j}\right. \\
& \left.+\tilde{Y}_{U}^{i j} \bar{U}_{L}^{i} \mathcal{H}_{b a}^{*}\left(L_{U, R}^{j}\right)_{a b}+\text { h.c }\right]+(U \rightarrow D), \\
& \mathcal{L}_{\text {mix }}^{\text {bidoublet }}=\lambda_{L u}^{i j} \bar{q}_{L a}^{i} Q_{R u a}^{j}+\lambda_{R u}^{i j} \bar{U}_{L}^{i} u_{R}^{j}+(U, u \rightarrow D, d),
\end{aligned}
$$

where $L_{U}$ transforms as a $(\mathbf{2}, \mathbf{2})_{2 / 3}$ and $L_{D}$ transforms as a $(\mathbf{2}, \mathbf{2})_{-1 / 3}$ under the composite gauge group. $U$ and $D$ are singlets with the $U(1)_{X}$ charge $2 / 3$ and $-1 / 3$, respectively. The components of the multiplets are

$$
\begin{aligned}
& L_{U}=\left(\begin{array}{ll}
Q_{u} & Q_{u}^{\prime}
\end{array}\right)=\left(\begin{array}{ll}
T & T_{5 / 3} \\
B & T_{2 / 3}
\end{array}\right), \\
& L_{D}=\left(Q_{d}^{\prime} Q_{d}\right)=\left(\begin{array}{ll}
B_{-1 / 3} & T^{\prime} \\
B_{-4 / 3} & B^{\prime}
\end{array}\right) .
\end{aligned}
$$

After rotating to the mass basis, the light and mostly elementary SM fermions couple to the Higgs through their mixings $\lambda$ with the composite states. For example, in the doublet model, the mass matrix of light quarks, after removing the mixing with the heavy fermions but before rotating to the mass basis, can be written as

$\left(m_{u, d}\right)_{i j}=\frac{v}{\sqrt{2}}\left(\lambda_{L} m_{Q}^{-1} Y m_{R}^{-1} \lambda_{R u, d}\right)_{i j}+O\left(\frac{v^{3}}{m_{Q, R}^{3}}\right)$,

where $v=246 \mathrm{GeV}$ is the Higgs vacuum expectation value, and similar expressions hold in the bidoublet and triplet models. At leading order in $v / m_{Q, R}$, only the Yukawa couplings $Y$ (and not $\tilde{Y}$ ) enter the mass matrix, which is why the latter are sometimes called "wrong-chirality" Yukawa couplings. Although they are not necessary for the generation of quark masses, they are present in many models, and we will see that they play a crucial role in the generation of dipole operators, so we keep them in our Lagrangians.

\section{Model-independent phenomenology of dipole operators}

\subsection{Effective Hamiltonian}

We are interested in the electromagnetic and chromomagnetic dipole operators involving quarks, both flavour violating and flavour conserving. The relevant effective Hamiltonian can be written as

$$
\mathcal{H}_{\mathrm{eff}}=-\sum_{i, j, q, V} C_{q_{i} q_{j} V} Q_{q_{i} q_{j} V}+C_{q_{i} q_{j} V}^{\prime} Q_{q_{i} q_{j} V}^{\prime},
$$

where $q=u, d$ and $V=\gamma, g$. We define the dipole operators as

$$
\begin{aligned}
Q_{q_{i} q_{j} \gamma} & =\frac{e m_{q_{i}}}{16 \pi^{2}}\left(\bar{q}_{j} \sigma^{\mu \nu} P_{R} q_{i}\right) F_{\mu \nu}, \\
Q_{q_{i} q_{j} g} & =\frac{g_{s} m_{q_{i}}}{16 \pi^{2}}\left(\bar{q}_{j} \sigma^{\mu \nu} T^{a} P_{R} q_{i}\right) G_{a \mu \nu}, \\
Q_{q_{i} q_{j} \gamma}^{\prime} & =\frac{e m_{q_{i}}}{16 \pi^{2}}\left(\bar{q}_{j} \sigma^{\mu \nu} P_{L} q_{i}\right) F_{\mu \nu}, \\
Q_{q_{i} q_{j} g}^{\prime} & =\frac{g_{s} m_{q_{i}}}{16 \pi^{2}}\left(\bar{q}_{j} \sigma^{\mu \nu} T^{a} P_{L} q_{i}\right) G_{a \mu \nu} .
\end{aligned}
$$

In the flavour-conserving case, one has $C_{q q V}^{\prime}=C_{q q V}^{*}$, so in total there are 18 magnetic and 18 chromomagnetic quark dipole operators. Among those, the most phenomenologically relevant ones are the first-generation flavour-conserving operators contributing to the neutron EDM, the flavourviolating ones with down-type quarks contributing to FCNCs with $B$ and $K$ mesons, as well as $Q_{c u V}^{(\prime)}$ relevant for charm FCNCs. Before discussing the observables probing these operators in turn, we briefly summarise the QCD evolution that is necessary to relate the operators generated at a high new physics scale to the low-energy observables.

\subsection{QCD corrections}

The operators $O_{q_{i} q_{j} \gamma}^{(\prime)}$ and $O_{q_{i} q_{j} g}^{(\prime)}$ are subject to QCD renormalisation and undergo mixing. They evolve according to (omitting flavour indices)

$$
\left(\begin{array}{l}
C_{\gamma}\left(\mu_{l}\right) \\
C_{g}\left(\mu_{l}\right)
\end{array}\right)=\left(\begin{array}{cc}
\eta_{\gamma \gamma} & \eta_{\gamma g} \\
0 & \eta_{g g}
\end{array}\right)\left(\begin{array}{l}
C_{\gamma}\left(\mu_{h}\right) \\
C_{g}\left(\mu_{h}\right)
\end{array}\right),
$$

and equivalently for the primed coefficients. For the running from some high new physics matching scale $\mu_{h}$ down to the top mass $m_{t}$, one has at leading logarithmic order [36]

$$
\begin{aligned}
& \eta_{\gamma \gamma}=\left[\frac{\alpha_{s}\left(\mu_{h}\right)}{\alpha_{s}\left(m_{t}\right)}\right]^{16 / 21}, \quad \eta_{g g}=\left[\frac{\alpha_{s}\left(\mu_{h}\right)}{\alpha_{s}\left(m_{t}\right)}\right]^{2 / 3}, \\
& \eta_{\gamma g}=\frac{8}{3}\left(\left[\frac{\alpha_{s}\left(\mu_{h}\right)}{\alpha_{s}\left(m_{t}\right)}\right]^{2 / 3}-\left[\frac{\alpha_{s}\left(\mu_{h}\right)}{\alpha_{s}\left(m_{t}\right)}\right]^{16 / 21}\right) .
\end{aligned}
$$

For the evolution from $m_{t}$ down to some low scale $\mu_{l}$, the number of active quark flavours change and quark mass thresholds have to be taken into account. We list numerical values of the evolution coefficients $\eta$ for the evolution from some exemplary high scale values to $m_{t}$, as well as from $m_{t}$ to phenomenologically relevant low scales, in Table 1.

In writing Eq. (13), we have neglected the mixing of neutral or charged current-current (four-quark) operators into the dipole operators [37]. Although such operators are generated at tree level in our setup, we expect that their contributions to the dipole operators are small, since they are suppressed by additional powers of the composite-elementary mixing angles. 
Table 1 RG coefficients for the evolution from some high new physics scale $\mu_{h}$ to $m_{t}$ (left), and from $m_{t}$ to some low-energy scale $\mu_{l}$. We use $\alpha_{s}\left(M_{Z}\right)=0.1185$

\begin{tabular}{llllllll}
\hline$\mu_{h}(\mathrm{TeV})$ & $\eta_{\gamma \gamma}$ & $\eta_{\gamma g}$ & $\eta_{g g}$ & $\mu_{l}$ & $\eta_{\gamma \gamma}$ & $\eta_{\gamma g}$ & $\eta_{g g}$ \\
\hline 0.5 & 0.905 & 0.030 & 0.917 & $m_{W}$ & 0.930 & 0.023 & 0.939 \\
1 & 0.856 & 0.045 & 0.873 & $m_{b}$ & 0.603 & 0.105 & 0.642 \\
2 & 0.813 & 0.057 & 0.835 & $2 \mathrm{GeV}$ & 0.502 & 0.120 & 0.547 \\
5 & 0.763 & 0.070 & 0.789 & $m_{c}$ & 0.432 & 0.127 & 0.480 \\
& & & & $1 \mathrm{GeV}$ & 0.389 & 0.130 & 0.438 \\
\hline
\end{tabular}

\subsection{Neutron EDM}

The electric and chromoelectric dipole moments (EDMs and CEDMs) of the quarks are related to the Wilson coefficients of the flavour-conserving dipole operators as

$d_{q}=\frac{e m_{q}}{8 \pi^{2}} \operatorname{Im}\left(C_{q q \gamma}\left(\mu_{l}\right)\right), \quad \tilde{d}_{q}=\frac{g_{s} m_{q}}{8 \pi^{2}} \operatorname{Im}\left(C_{q q g}\left(\mu_{l}\right)\right)$,

where $\mu_{l}$ is a hadronic scale of order $1 \mathrm{GeV}$. The calculation of the contributions of the quark (C)EDMs to the neutron EDM is plagued by considerable hadronic uncertainties. An estimate obtained using QCD sum rules [38] yields

$d_{n}=(1 \pm 0.5)\left[1.4\left(d_{d}-\frac{1}{4} d_{u}\right)+1.1 e\left(\tilde{d}_{d}+\frac{1}{2} \tilde{d}_{u}\right)\right]$.

Experimentally, the neutron EDM is already strongly constrained [39],

$\left|d_{n}\right|<2.9 \times 10^{-26} e \mathrm{~cm}$ at $90 \%$ C.L.

Several experiments are in construction that plan to improve this bound by up to two orders of magnitude [40].

Indirectly, the neutron EDM is also sensitive to the CEDMs of second- and third-generation quarks. In the QCD evolution of the CEDMs to low energies, when integrating out a heavy quark, a finite threshold correction is generated to the three-gluon Weinberg operator, which directly contributes to the neutron EDM and mixes under renormalisation with the first-generation quark (C)EDMs [41]. Taking these effects into account, the bound (17) can be translated into bounds on the charm, bottom and top CEDMs, which read [42-44]

$$
\begin{aligned}
\left|\tilde{d}_{c}\right| & <1.0 \times 10^{-22} \mathrm{~cm}, \\
\left|\tilde{d}_{b}\right| & <1.1 \times 10^{-21} \mathrm{~cm}, \\
\left|\tilde{d}_{t}\right| & <2.1 \times 10^{-19} \mathrm{~cm} .
\end{aligned}
$$

\subsection{Down-type FCNCs}

The most well-measured flavour-changing dipole transitions are the $b \rightarrow s \gamma / g$ processes probed in the inclusive decay $B \rightarrow X_{s} \gamma$. The corresponding decay probing the $b \rightarrow d \gamma / g$ transitions is even rarer in the SM due to the stronger CKM suppression and consequently is measured less precisely. Normalizing the current experimental measurements to the SM expectations for the branching ratios,

$R_{b q \gamma}=\frac{\mathrm{BR}\left(B \rightarrow X_{q} \gamma\right)}{\mathrm{BR}\left(B \rightarrow X_{q} \gamma\right)_{\mathrm{SM}}}, \quad$ with $q=s, d$,

one has at present [45-48]

$R_{b s \gamma}=1.13 \pm 0.11, \quad R_{b d \gamma}=0.92 \pm 0.40$.

Beyond the SM, these quantities are modified as $[37,49]$

$R_{b q \gamma}=1+0.97\left(2 \operatorname{Re}\left(R_{7 q}\right)+\left|R_{7 q}\right|^{2}+\left|R_{7 q}^{\prime}\right|^{2}\right)$,

where

$R_{7 q}^{(\prime)}=\frac{\sqrt{2}}{4 G_{F} V_{t b} V_{t q}^{*}} \frac{C_{b q \gamma}^{(\prime)}\left(m_{b}\right)}{C_{7}^{\text {eff }}\left(m_{b}\right)}$,

with $C_{7}^{\text {eff }}\left(m_{b}\right)=-0.3523$. For the numerical bounds on the Wilson coefficients in the next sections, we imposed the constraints (20) at $2 \sigma$.

The $s \rightarrow d \gamma / g$ transitions are less constrained experimentally, since the long-distance dominance in $K$ decay processes makes it difficult to relate experimental observables to the short-distance contributions. Nevertheless, a meaningful bound on the Wilson coefficients $C_{s d g}^{(\prime)}$ can be obtained from the measurement of the parameter $\epsilon^{\prime} / \epsilon$. With the conservative assumption that the new physics contribution to $\epsilon^{\prime} / \epsilon$ should not exceed its experimental central value, one obtains the bound [50]

$\frac{1}{2} \operatorname{Im}\left(C_{s d g}-C_{s d g}^{\prime}\right)<3.1 \times 10^{-8}$.

\subsection{Charm FCNCs}

Recent experimental hints that the direct $\mathrm{CP}$ asymmetry difference $\Delta A_{\mathrm{CP}}$ between $D \rightarrow K K$ and $D \rightarrow \pi \pi$ decays is larger than the SM expectation have attracted a lot of interest as a possible sign of new physics, also in the context of models with partial compositeness [25,51]. But even if the observed effect is not due to new physics, the upper bound on $\Delta A_{\mathrm{CP}}$ can be used to put a constraint on the charm chromomagnetic dipole operator $Q_{\text {cug }}^{\prime}$ [52]. Following [44], we impose in the numerical analysis that the new physics contribution 
Table 2 Model-independent bounds on new physics contributions to Wilson coefficients of dipole operators. The four columns show the lower bounds on $M$, where the Wilson coefficients at the matching scale of $1 \mathrm{TeV}$ were assumed to be $C_{q_{i} q_{j} V}(1 \mathrm{TeV})=$ $(1,-1, i,-i) / M^{2}$

\begin{tabular}{|c|c|c|c|c|c|}
\hline Operator & $\begin{array}{l}\operatorname{Re}(C)<M^{-2} \\
(\mathrm{TeV})\end{array}$ & $\begin{array}{l}\operatorname{Re}(C)>-M^{-2} \\
(\mathrm{TeV})\end{array}$ & $\begin{array}{l}\operatorname{Im}(C)<M^{-2} \\
(\mathrm{TeV})\end{array}$ & $\begin{array}{l}\operatorname{Im}(C)>-M^{-2} \\
(\mathrm{TeV})\end{array}$ & Process \\
\hline$Q_{u u \gamma}$ & & & 1.08 & & $d_{n}$ \\
\hline$Q_{d d \gamma}$ & & & 3.11 & & $d_{n}$ \\
\hline$Q_{\text {uиg }}$ & & & 1.45 & & $d_{n}$ \\
\hline$Q_{d d g}$ & & & 3.79 & & $d_{n}$ \\
\hline$Q_{c c g}$ & & & 1.22 & & $d_{n}$ \\
\hline$Q_{b b g}$ & & & 0.67 & & $d_{n}$ \\
\hline$Q_{t t g}$ & & & 0.30 & & $d_{n}$ \\
\hline$Q_{b s \gamma}$ & 0.71 & 2.81 & 1.44 & 1.39 & $B \rightarrow X_{s} \gamma$ \\
\hline$Q_{b s g}$ & 0.34 & 1.34 & 0.69 & 0.67 & $B \rightarrow X_{s} \gamma$ \\
\hline$Q_{b s \gamma}^{\prime}$ & 1.41 & & 1.31 & & $B \rightarrow X_{s} \gamma$ \\
\hline$Q_{b s g}^{\prime}$ & 0.68 & & 0.68 & & $B \rightarrow X_{s} \gamma$ \\
\hline$Q_{b d \gamma}$ & 3.74 & 1.51 & 2.91 & 1.94 & $B \rightarrow X_{d} \gamma$ \\
\hline$Q_{b d g}$ & 1.79 & 0.72 & 1.40 & 0.93 & $B \rightarrow X_{d} \gamma$ \\
\hline$Q_{b d \gamma}^{\prime}$ & 2.37 & & 2.37 & & $B \rightarrow X_{d} \gamma$ \\
\hline$Q_{b d g}^{\prime}$ & 1.14 & & 1.14 & & $B \rightarrow X_{d} \gamma$ \\
\hline$Q_{s d g}^{(\prime)}$ & & & 2.80 & & $\epsilon^{\prime} / \epsilon$ \\
\hline$Q_{\text {cug }}^{(\prime)}$ & & & 2.14 & & $D \rightarrow K K, \pi \pi$ \\
\hline
\end{tabular}

to $\Delta A_{\mathrm{CP}}$, for central values of the hadronic parameters, does not exceed the world average [45]

$\Delta A_{\mathrm{CP}}=(-0.319 \pm 0.121) \%$.

\subsection{Model-independent bounds}

Given all the experimental constraints discussed above, we can derive model-independent bounds on the Wilson coefficients of the dipole operators. We list them in Table 2 at a renormalisation scale of $1 \mathrm{TeV}$, considering one purely real or purely imaginary Wilson coefficient at a time.

The only operators in the effective Hamiltonian (10) we have not considered are the flavour-changing ones involving top quarks. Although they are not yet strongly constrained, they will be probed at LHC in the future through the decays $t \rightarrow q \gamma$ and $t \rightarrow q g$, where $q=u, c$.

\section{Analytical results for the Wilson coefficients}

In this section, we derive approximate analytical expressions for the Wilson coefficients of the dipole operators for the three different choices of fermion representations. We denote by $M \sim m_{Q, R}$ a generic composite mass, by $\lambda$ a generic composite-elementary mixing parameter, by $g$ an elementary gauge coupling and by $g_{\rho}$ the coupling of the composite vector resonances. Our goal is to obtain expressions for the Wilson coefficient to a given order in the small ratios $v / M, \lambda / M$, and $g / g_{\rho}$. To this end, we first consider the case of a single generation of elementary and composite fermions. The relevant mass matrices arising in the three models are collected in Appendix 8. We diagonalise these matrices at a given order in the small ratios, rotate all couplings to the mass eigenstate basis and compute the Wilson coefficients. The resulting oneloop expressions for the contributions to the Wilson coefficients involving scalars or vectors are listed along with the relevant loop functions in Appendix 9. It turns out that the dominant contributions typically arise from diagrams with a heavy fermion-lifting the chirality suppression-together with a $W, Z$ or Higgs in the loop. In Sect. 4.1, we first discuss these contributions in detail, before qualitatively discussing the additional contributions in Sect. 4.2.

\subsection{Leading contributions}

For a single generation of fermions, to leading order in the small parameters $v / M, \lambda / M$ and in the limit of heavy vector resonances, we find that the Wilson coefficients can be written in the form ${ }^{3}$

$C_{q q V}=C_{q q V}^{\mathrm{SM}}+a_{q V} \frac{Y \tilde{Y}}{m_{Q^{m}}}$,

where $q=u, d$ and $V=\gamma, g$. In this limit, the only relevant diagrams feature a Higgs, $W$ or $Z$ boson as well as a heavy

3 Here and in the following, to simplify the notation, we will assume $m_{Q_{u}}=m_{Q_{d}} \equiv m_{Q}, m_{U}=m_{D} \equiv m_{R}, Y_{U}=Y_{D} \equiv Y$ and $\tilde{Y}_{U}=$ $\tilde{Y}_{D} \equiv \tilde{Y}$ in the bidoublet model. 
Table 3 Coefficients entering the leading-order contribution (25) to the dipole Wilson coefficients of down-type quarks (left) and up-type quarks (right)

\begin{tabular}{llllllll}
\hline & Doublet & Triplet & Bidoublet & & Doublet & Triplet & Bidoublet \\
\hline$a_{d \gamma}$ & $\frac{1}{4}$ & $\frac{1}{2}$ & $-\frac{1}{2}$ & $a_{u \gamma}$ & 0 & $\frac{10}{3}$ & 1 \\
$a_{d g}$ & $\frac{3}{4}$ & $\frac{3}{2}$ & $\frac{3}{2}$ & $a_{u g}$ & $\frac{3}{4}$ & 2 & $\frac{3}{2}$ \\
\hline
\end{tabular}

fermion in the loop. We have computed all coefficients $a_{q V}$ in the doublet, triplet and bidoublet models and list them in Table 3. To illustrate our procedure, we give a detailed account of our calculation of $a_{d \gamma}$ in the bidoublet model in Appendix 10. We note that the value $a_{u \gamma}=0$ in the doublet model is not due to a symmetry but rather due to an accidental cancellation between the $W, Z$ and Higgs contributions.

An important result of our calculation is that, at leading order, there is no quadratic term in $Y$, as was also emphasised in Ref. [25] in the context of the $c \rightarrow u g$ dipole transition ${ }^{4}$. This means that in models in which the "wrong-chirality" Yukawa couplings are absent or suppressed, the dipole operators will be suppressed as well. We will discuss other contributions, which become the leading ones in the limit $\tilde{Y} \rightarrow 0$, in Sect. 4.2.

The result in Eq. (25) is only valid in the unrealistic case of a single generation of fermions. Taking into account all three generations and an arbitrary flavour structure, the full analytic diagonalisation of the mass matrices is clearly not feasible. Still, it is possible to obtain an approximate analytical expression valid for three generations of elementary and composite quarks by promoting Eq. (25) to a matrix equation in flavour space. Concretely, for $i \geq j$, one has

$C_{d_{i} d_{j} V}=C_{d_{i} d_{j} V}^{\mathrm{SM}}+\frac{a_{d V}}{m_{d_{i}}} \Delta_{j i}^{d}$,

$C_{d_{i} d_{j} V}^{\prime}=\frac{a_{d V}}{m_{d_{i}}} \Delta_{i j}^{d}$,

where $\Delta^{d}=\frac{v}{\sqrt{2}} U_{L d}^{\dagger} \lambda_{L} m_{Q}^{-1} Y m_{R}^{-1} \tilde{Y} m_{Q}^{-1} Y m_{R}^{-1} \lambda_{R d} U_{R d}$,

and analogously for up-type quarks, ${ }^{5}$ where $U_{L q, R q}$ are the matrices diagonalizing the quark mass terms (9). We checked numerically that Eqs. (26)-(28) indeed give a very good approximation to the exact results obtained by numerically diagonalizing the mass matrices.

\footnotetext{
4 The proportionality to the wrong-chirality Yukawa was also found for the $\mu \rightarrow e \gamma$ dipole in Ref. [53].

5 In the triplet model, there is an additional factor of $\frac{1}{\sqrt{2}}$ in front of $\Delta^{u}$ compared to Eqs. (26)-(28), cf. the mass matrices in Appendix 8.
}

\subsection{Subleading contributions}

Going beyond the leading order in the expansion of composite-elementary mixings and $v / M$ and beyond the limit of heavy vector resonances, there are several classes of contributions that can become relevant in some cases, in particular in models without wrong-chirality Yukawa couplings. This can be the case e.g. in Randall-Sundrum models with the Higgs field strictly localised on the IR brane, where the presence of wrong-chirality Yukawa couplings is not required by $5 \mathrm{D}$ gauge invariance (see e.g. [54]). In general, no simple analytical expressions can be given for these subleading contributions, so our discussion will remain qualitative.

\subsubsection{Higher orders in elementary-composite mixing}

These contributions are suppressed by $\lambda^{2} / M^{2}$ with respect to Eq. (25) and are relevant for Wilson coefficients involving the third generation, which can have a sizable degree of compositeness, in particular for $b \rightarrow q \gamma / g$. These contributions can arise

(a) from diagrams with a SM quark and a $W$ or $Z$ in the loop and an $O\left(v^{2} / M^{2}\right)$ correction to the quark-gauge boson vertex;

(b) from diagrams with a heavy fermion and a $W, Z$, or Higgs in the loop that are parametrically of the same order.

We start by discussing the contributions of type (a). In the case of $b \rightarrow s \gamma / g$ and $b \rightarrow d \gamma / g$, there are two contributions to the $W$-top loop that are only suppressed by the degree of compositeness of the right-handed top quark compared to the leading contribution. They read

$\begin{aligned} \delta C_{b q V} & =\frac{4 G_{F}}{\sqrt{2}} \frac{m_{t}}{m_{b}} V_{t q}^{*}\left(\delta g_{W}^{R}\right)_{t b} f_{V}\left(x_{t}\right), \\ \delta C_{b q V}^{\prime} & =\frac{4 G_{F}}{\sqrt{2}} \frac{m_{t}}{m_{b}} V_{t b}\left(\delta g_{W}^{R}\right)_{t q} f_{V}\left(x_{t}\right),\end{aligned}$

where $q=d, s, x_{t}=m_{t}^{2} / m_{W}^{2}$ and the $W$ coupling of the SM quarks is written as

$\mathcal{L} \supset \frac{g}{\sqrt{2}} \bar{u}_{i}\left[\left(V_{i j}+\left(\delta g_{W}^{L}\right)_{i j}\right) \gamma^{\mu} P_{L}+\left(\delta g_{W}^{R}\right)_{i j} \gamma^{\mu} P_{R}\right] d_{j} W_{\mu}^{+}$.

In general, one has

$$
\begin{aligned}
& \left(\delta g_{W}^{L}\right)_{i j}=a v^{2}\left(\lambda_{L} m_{Q}^{-1} Y Y^{\dagger} m_{Q}^{-1} \lambda_{L}\right)_{i j}, \\
& \left(\delta g_{W}^{R}\right)_{i j}=b v^{2}\left(\lambda_{R u} m_{R}^{-1} Y^{\dagger} Y m_{R}^{-1} \lambda_{R d}\right)_{i j},
\end{aligned}
$$

where the coefficients $a$ and $b$ are given in Table 4 . 
Table 4 Coefficients in Eq. (32) relevant for the corrections to the $W$ couplings in all models

\begin{tabular}{llll}
\hline & Doublet & Triplet & Bidoublet \\
\hline$a$ & $-\frac{1}{2}$ & $-\frac{1}{4}$ & $-\frac{1}{4}$ \\
$b$ & $\frac{1}{2}$ & $\frac{\sqrt{2}}{4}$ & 0 \\
\hline
\end{tabular}

The contribution in Eq. (29) due to the right-handed Wtb coupling was first discussed in Ref. [26]. We emphasise that the contribution in Eq. (30) can be equally important in specific models, although it depends on the flavour structure of $\delta g_{W}^{R}$, while the contribution in Eq. (29) is present even for a flavour diagonal $\delta g_{W}^{R}$. Concerning the contributions of type (b), which involve heavy fermions in the loop, we merely note that they are parameterically of the same order as the ones of type (a), but can have a different flavour structure and are therefore more model dependent. Contributions involving the degree of compositeness of the left-handed bottom quark are suppressed by $m_{b} / m_{t}$, and we will neglect them.

\subsubsection{Higher orders in inverse powers of the composite mass scale}

We now consider terms that do not involve additional composite-elementary mixings, but are present even in the limit $\tilde{Y} \rightarrow 0$. These contributions are relevant for Wilson coefficients not involving the third generation in models where the "wrong-chirality" Yukawas $\tilde{Y}$ are absent or suppressed. Such contributions arise for example from an expansion of the loop functions of the diagrams with a $W$, $Z$ or Higgs and a heavy fermion to higher order in the ratios $x=m_{\psi}^{2} / m_{W, Z, h}^{2}$, where $m_{\psi}$ is the heavy fermion mass. In fact, the analytic cancellation of the contributions proportional to $Y^{2}$ works only at the leading order of the expansion of the loop functions. As an example, we discuss the Higgs contribution to the down-type quark dipole operator for a single generation in the bidoublet model for $\tilde{Y}=0$. We find

$C_{d d V} \supset-\frac{1}{6} \frac{m_{h}^{2}\left(m_{Q}^{2}+m_{R}^{2}\right) Y^{2}}{m_{Q}^{3} m_{R}^{3}}$.

There are similar contributions suppressed by $m_{W}^{2} / m_{\psi}^{2}$ and $m_{Z}^{2} / m_{\psi}^{2}$. In the case of the $W$ contribution, there is the special feature that, in addition to the quadratic term in the mass ratio, there is also a logarithm that becomes dominant for large fermion resonance mass. In view of this complicated dependence, we refrain from giving full analytical expressions for this type of subleading contributions in all models, but simply keep in mind that, in the absence of wrong-chirality Yukawa couplings and sizable compositeelementary mixings, the Higgs, $W$ and $Z$ contributions to

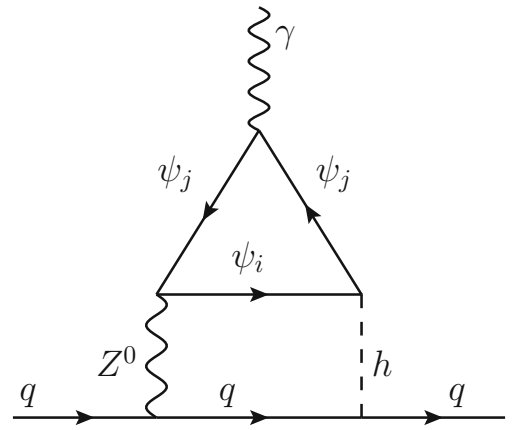

Fig. 1 Two-loop Barr-Zee-type diagram contributing to the electromagnetic dipole operator

the dipole operators are roughly suppressed by $m_{h, W, Z}^{2} / m_{\psi}^{2}$ compared to the leading contribution for non-zero $\tilde{Y}$.

\subsubsection{Higher loop orders}

Two-loop contributions to the dipole operators might be relevant in cases where the wrong-chirality Yukawas are absent or strongly suppressed, operators not involving the third generation are considered (in particular, EDMs), and the composite mass scale is large. For operators involving the third generation, the contributions discussed in Sect. 4.2.1 dominate instead. The last item is relevant because the contributions discussed in Sect. 4.2.2 decouple with the fourth power of the inverse mass scale, while at two-loop order, there can be diagrams that decouple with the square of the inverse mass scale, but that do not vanish for $\tilde{Y}=0$. An example is given by the Barr-Zee-type diagram shown in Fig. 1, which is familiar from the two-loop chargino contribution to the EDM in split supersymmetry [55]. We estimate the contribution of this diagram to the Wilson coefficient in the limit $\tilde{Y}=0$ as

$C_{q_{i} q_{j} V} \sim \frac{g^{2}}{16 \pi^{2}} \frac{Y^{2}}{m_{\psi}^{2}}$

up to an $O(1)$ factor. We see that it can be safely neglected with respect to the leading contribution (25) even for $\tilde{Y} \sim$ $Y$, but it can dominate compared to the contribution (33) if $m_{\psi} \sim m_{Q} \sim m_{R}$ is in the multi-TeV regime.

\subsubsection{Diagrams with heavy vector resonances}

Until now, we have only considered one-loop diagrams with a heavy fermion and a $W, Z$ or Higgs in the loop, but there are also diagrams with a heavy vector resonance and a heavy fermion. These contributions are always parametrically suppressed by a factor $g_{\rho}^{2} / m_{\rho}^{2}$, where $g_{\rho}$ is the coupling and $m_{\rho}$ the mass of the vector resonance. In general, the analytical expressions for these contributions are complicated, since, in contrast to the $W$ or $Z$ contributions considered above, 
one has to keep the full dependence of the loop functions if the fermion and vector resonance masses are comparable. However, it is important to notice that in the limit where all the fermion resonances are degenerate, the contribution to the dipole operators from these diagrams is real and diagonal in the mass basis and thus does not contribute to any of the observables we consider, which always feature either flavour or $\mathrm{CP}$ violation. ${ }^{6}$ In general, we find that in the case of non-zero $\tilde{Y}$, these contributions are always suppressed by $v^{2} g_{\rho}^{2} / m_{\rho}^{2}$ and/or $m_{\psi}^{2} / m_{\rho}^{2}$ (which is preferred to be smaller than 1 since naturalness prefers light fermion resonances and electroweak precision tests require heavy vector resonances) with respect to the leading contribution (25), and we confirmed with a numerical scan that they are typically small. We will not consider this class of contributions in the following, but one should keep in mind that, in particular corners of the parameter space, they might be relevant in specific models and would tighten the bounds considered below.

\subsubsection{Higher-dimensional operators}

Finally, in a more complete theory like a composite Higgs model, there can be additional contributions that are not captured by our Lagrangians defined at the beginning of Sect. 2 and are therefore not calculable in our setup. This means that the bounds we obtain below can be viewed as conservative estimates. It is possible that there are additional contributions that make the bounds more severe; but, on general grounds, there is no reason to expect that these additional effects conspire with the calculable ones to eliminate the constraints.

\section{Phenomenological analysis}

We now proceed to a numerical analysis of the bounds on partial compositeness from observables sensitive to dipole operators. Since all these observables probe either flavour or $\mathrm{CP}$ violation (or both), the bounds crucially depend on the assumptions made on the flavour structure of the model. We start with the most popular assumption of flavour anarchy, which arises in models aiming at a geometrical explanation for the quark mass and mixing hierarchies but is known to have a problem (assuming $\mathrm{TeV}$-scale resonance masses) with excessive $\mathrm{CP}$ violation in $K$ mixing, unless one is willing to accept an $O\left(10^{-2}\right)$ fine tuning of the relevant CP-violating phase. We then also consider models with a global flavour symmetry in the strong sector-either $U(3)^{3}$ or $U(2)^{3}$ —only broken by the composite-elementary mixings.

\footnotetext{
${ }^{6}$ In the lepton sector, which we do not consider here, the muon anomalous magnetic moment is an important exception, because it does not require any flavour or $\mathrm{CP}$ violation.
}

Our aim in this section is not to perform a full numerical analysis of these models and the contributions to dipole operators. Rather, we aim to provide analytical expressions for the dominant contributions to the dipole operators and use them to extract approximate lower bounds on the resonance masses from the experimental measurements. These results can then be used to judge how severely a model with a given choice of fermion representations and with a given flavour structure is constrained by the observables sensitive to dipole operators.

\subsection{Models with flavour anarchy}

We first consider the case of flavour anarchy [13-19], where all the couplings in the strong sector are assumed to have $O$ (1) off-diagonal elements and phases. In general, all coefficients then depend on complicated functions of the anarchic Yukawa and mass matrix elements. To give simplified approximate expressions one can use the fact that, up to $O(1)$ factors, the quark Yukawa couplings and the CKM matrix elements can be written in terms of the degrees of compositeness $s_{L, R}$ (see Appendix 8 for their definition) as

$y_{u_{i}} \sim Y s_{L i} s_{R u_{i}}, \quad y_{d_{i}} \sim Y s_{L i} s_{R d_{i}}, \quad V_{i j} \sim s_{L i} / s_{L j} \quad(j>i)$.

where $Y$ can be understood as an "average" Yukawa coupling. In the following, we provide simplified expressions for the Wilson coefficients in terms of "average" parameters $Y, \tilde{Y}$ and $m_{i}$ that keep track of how the quantities scale with the parameters, but we neglect $O(1)$ factors coming from the flavour structure. We do, however, take into account the numerical factors derived in Sect. 4.

The leading contributions to the Wilson coefficients at the matching scale then read

$$
\begin{aligned}
C_{q q V} & \sim a_{q V} \frac{Y \tilde{Y}}{m_{Q} m_{R}} \quad \text { for } q=u, d, \\
C_{b q V} & \sim V_{t q} a_{d V} \frac{Y \tilde{Y}}{m_{Q} m_{R}} \quad \text { for } q=d, s, \\
C_{b q V}^{\prime} & \sim \frac{m_{q}}{m_{b} V_{t q}} a_{d V} \frac{Y \tilde{Y}}{m_{Q} m_{R}} \quad \text { for } q=d, s, \\
C_{s d g} & \sim V_{c d} a_{d g} \frac{Y \tilde{Y}}{m_{Q} m_{R}}, \\
C_{s d g}^{\prime} & \sim \frac{m_{d}}{m_{s} V_{c d}} a_{d g} \frac{Y Y}{m_{Q} m_{R}}, \\
C_{c u g} & \sim V_{u s} a_{u g} \frac{Y \tilde{Y}}{m_{Q} m_{R}}, \\
C_{c u g}^{\prime} & \sim \frac{m_{u}}{m_{c} V_{u s}} a_{u g} \frac{Y \tilde{Y}}{m_{Q} m_{R}},
\end{aligned}
$$


Table 5 Lower bounds on the average fermion resonance mass (multiplied by a combination of parameters, as indicated in the first row) in flavour anarchic models with $\tilde{Y} \neq 0$ (first three columns), and crude estimates in the limit $\tilde{Y}=0$ (last two columns). The mass bounds get stronger for larger $Y$ and/or $\tilde{Y}$

\begin{tabular}{llllll}
\hline Bound on: & $\left(\frac{m_{Q} m_{R}}{Y \tilde{Y}}\right)^{1 / 2}$ & & & $\left(\frac{m_{Q}^{2}}{s_{R t}^{2} Y^{2}}\right)^{1 / 2}$ & $\left(\frac{m_{Q} m_{R}}{Y}\right)^{1 / 2}$ \\
\cline { 2 - 4 } Operator & Doublet (TeV) & Triplet (TeV) & Bidoublet (TeV) & (estimate) (TeV) & (estimate) (TeV) \\
\hline$Q_{d d V}$ & 3.6 & 5.1 & 4.1 & & 0.8 \\
$Q_{u u V}$ & 1.3 & 0.6 & 1.4 & & 0.3 \\
$Q_{c c g}$ & 1.1 & 1.7 & 1.5 & & 0.5 \\
$Q_{b b g}$ & 0.6 & 0.9 & 0.8 & 0.6 & 0.3 \\
$Q_{t t g}$ & 0.3 & 0.4 & 0.4 & 1.1 & 0.2 \\
$Q_{b s V}$ & 0.4 & 0.5 & 0.2 & 0.3 & 0.3 \\
$Q_{b s V}^{\prime}$ & 0.7 & 1.0 & 0.4 & 0.9 & 0.3 \\
$Q_{b d V}$ & 0.2 & 0.3 & 0.1 & & 0.3 \\
$Q_{b d V}^{\prime}$ & 0.6 & 0.8 & 0.3 & & 0.3 \\
$Q_{s d g}^{\prime}$ & 1.1 & 1.6 & 1.6 & & 0.5 \\
$Q_{s d g}^{\prime}$ & 1.1 & 1.6 & 1.6 & & 0.4 \\
$Q_{c u g}$ & 0.9 & 1.4 & 1.3 & 0.3 \\
$Q_{c u g}^{\prime}$ & 0.2 & 0.3 & 0.2 & & 0.2 \\
\hline
\end{tabular}

where $V=\gamma, g$. Arbitrary phases and $O(1)$ factors are understood in all cases. Concerning the relative importance of the primed and unprimed flavour-changing Wilson coefficients, it is interesting to note that in $b \rightarrow s$ and $b \rightarrow d$ transitions the flavour prefactor is an order of magnitude larger for the primed coefficients, so observables in $B$ decays sensitive to the primed Wilson coefficients, i.e. to right-handed flavour-changing neutral currents, are particularly promising in the anarchic model (see [56] for an overview of promising observables). For the $s \rightarrow d$ transition, the prefactors of primed and unprimed coefficients are comparable, and for the $c \rightarrow u$ transition the unprimed coefficient has a prefactor that is about a factor 30 larger than the unprimed one.

For the $b \rightarrow s$ and $b \rightarrow d$ transitions, there is an additional important contribution that is only suppressed by the degree of compositeness of the right-handed top quark, as discussed in Sect. 4.2. Here, we give only a crude parametric estimate of this contribution,

$$
\begin{aligned}
\delta C_{b q V} & \sim V_{t q} \frac{Y^{2}}{m_{Q}^{2}} s_{R t}^{2}, \\
\delta C_{b q V}^{\prime} & \sim \frac{m_{q}}{m_{b} V_{t q}} \frac{Y^{2}}{m_{Q}^{2}} s_{R t}^{2} .
\end{aligned}
$$

For all Wilson coefficients, there is in addition a subleading contribution not involving $\tilde{Y}$ that is parametrically suppressed by $m_{h, W, Z}^{2} /\left(m_{Q} m_{R}\right)$ compared to the leading one (for $\tilde{Y} \sim Y$ ), as discussed in Sect. 4.2.2.

Having fixed the parametric dependencies of the Wilson coefficients up to $O(1)$ factors, we can proceed to put numerical bounds on the combination $Y \tilde{Y} /\left(m_{Q} m_{R}\right)$ and the corresponding quantities for the subleading contributions. These bounds are listed in Table 5. We make the following observations:

- The strongest bounds come from the down quark (C)EDM and constrain the fermion resonance masses to be above 4-5 TeV for $Y \sim \tilde{Y} \sim 1$.

- For $Y \sim \tilde{Y} \sim 1$, there is a multitude of bounds in the ballpark of 1-2 TeV. Since these refer to operators with different phases and flavour structures, we conclude that it will be hard to avoid all of them by fortuitous cancellations, even if the bounds listed here are subject to $O(1)$ uncertainties. Consequently, if $Y \sim \tilde{Y}$, dipole operators alone imply that sub-TeV fermion resonances are borderline and require a $Y$ not much larger than $1 .^{7}$

- In models with $\tilde{Y}=0$, the bounds turn out to be quite mild and an anarchic flavour and CP structure is compatible with sub-TeV fermion resonances for $Y \lesssim 3$, if only constraints from dipole operators are considered.

\subsection{Flavour-symmetric models}

Since the flavour anarchic model is not only plagued by strong constraints from dipole operators but also from meson-antimeson mixing induced at tree level, it has been suggested that the strong sector is invariant under a flavour symmetry that is only broken by the composite-elementary mixings of one chirality. The simplest case is a $U(3)^{3}$ symmetry broken by the composite-elementary mixings of right-handed quarks ("left-handed compositeness") or of

\footnotetext{
7 In the anarchic case, we are only referring to the average fermion resonance masses. Individual resonances could still accidentally be much lighter without necessarily violating flavour bounds.
} 
left-handed quarks ("right-handed compositeness") [32-34]. Among the three models considered here, right-handed compositeness can only be realised in the bidoublet model, as it requires different mixings for left-handed up- and downtype quarks. While the $U(3)^{3}$ models successfully suppress FCNCs, they are strongly constrained by electroweak and dijet constraints, since they predict a significant degree of compositeness for one chirality of light quarks [30,34]. This problem is avoided in models with a $U(2)^{3}$ flavour symmetry in the strong sector, again broken only by one chirality of composite-elementary mixings $[30,35]$.

\subsubsection{EDM constraints in $U(2)^{3}$ and $U(3)^{3}$ models}

In $U(3)^{3}$ flavour models with left- or right-handed compositeness, the parameters in the strong Lagrangian are generation invariant, e.g. for the triplet model,

$$
\begin{aligned}
\left(m_{Q}\right)_{i j} & =m_{Q} \delta_{i j}, \quad\left(m_{R}\right)_{i j}=m_{R} \delta_{i j}, \quad(Y)_{i j}=Y \delta_{i j} \\
(\tilde{Y})_{i j} & =\tilde{Y} \delta_{i j}
\end{aligned}
$$

and analogously for the other models. It can be shown that in all models the only physical phases apart from the CKM phase reside in the wrong-chirality Yukawa couplings $\tilde{Y}$ [34].

In flavour models based on a $U(2)^{3}$ symmetry, one has instead

$m_{Q}=\operatorname{diag}\left(m_{Q}, m_{Q}, m_{Q 3}\right), \quad Y=\operatorname{diag}\left(Y, Y, Y_{3}\right)$,

etc. As a result, there is an additional phase in the compositeelementary mixings related to the flavour symmetry-breaking spurions, but in the strong sector it is true as well that the only physical phases can be chosen to be the ones of the $\tilde{Y}$ couplings, which can be different for the third and the first two generations. Below, we will adopt a phase convention where $Y$ is real.

Consequently, in both $U(3)^{3}$ and $U(2)^{3}$ models, there is a clear-cut prediction for the flavour-conserving firstgeneration Wilson coefficients relevant for (C)EDMs,

$C_{q q V}=a_{q V} \frac{Y \tilde{Y}}{m_{Q} m_{R}} \quad$ for $q=u, d$,

where in $U(2)^{3}$, the masses and Yukawa couplings refer to those of the first two generations of composite fermions. Note that, in contrast to the anarchic model above, we have used a "=" sign, since there is no further $O(1)$ factor in front. This leads to the bounds on the combination $\frac{Y \operatorname{Im} \tilde{Y}}{m_{Q} m_{R}}$ shown in Table 6. We conclude that sub- $\mathrm{TeV}$ fermion resonances in $U(3)^{3}$ models, or sub-TeV fermion resonances of the first two generations in $U(2)^{3}$ models, require

$Y \operatorname{Im} \tilde{Y} \lesssim 0.05$
Table 6 Bounds from the neutron EDM on the quantity $\sqrt{m_{Q^{m_{R}}}} / \sqrt{Y \operatorname{Im} \tilde{Y}}$ in $U(3)^{3}$ and $U(2)^{3}$ models

\begin{tabular}{llll}
\hline Operator & Doublet $(\mathrm{TeV})$ & Triplet $(\mathrm{TeV})$ & Bidoublet $(\mathrm{TeV})$ \\
\hline$Q_{d d V}$ & 3.6 & 5.1 & 4.1 \\
$Q_{u u V}$ & 1.3 & 0.6 & 1.4 \\
$Q_{c c g}$ & 1.1 & 1.7 & 1.5 \\
$Q_{b b g}$ & 0.6 & 0.9 & 0.8 \\
$Q_{t t g}$ & 0.3 & 0.4 & 0.4 \\
\hline
\end{tabular}

As discussed above, in the limit $\tilde{Y} \rightarrow 0$ the strong sector carries no new phase both in $U(3)^{3}$ and $U(2)^{3}$ models. The remaining contributions to the EDMs involving the phases in the composite-elementary mixings are tiny, and hence there is no relevant bound.

In the case of the $U(2)^{3}$ model, if $\tilde{Y}=0$ or the firstgeneration fermion partners are decoupled, the leading contribution to the up- and down quark (C)EDMs is absent. But also the third-generation wrong-chirality Yukawa $\tilde{Y}_{3}$ can contribute to the neutron EDM, if it is complex. On the one hand, it will generate a contribution to the top CEDM via the Wilson coefficient

$C_{t t g}=a_{u g} \frac{Y_{3} \tilde{Y}_{3}}{m_{Q 3} m_{R 3}}$,

which leads to the bound shown in the last row of Table 6. On the other hand, a two-loop contribution to the first-generation EDMs proportional to $\operatorname{Im}\left(\tilde{Y}_{3}\right)$ can arise, e.g. from the diagram in Fig. 1. Estimating this contribution naively as $C_{q q V} \sim$ $g^{2} Y \tilde{Y} /\left(16 \pi^{2} m_{\psi}^{2}\right)$, one would obtain a similar bound on $m_{\psi}$ of the order of $0.4 \mathrm{TeV}$ for $Y_{3} \sim \operatorname{Im}\left(\tilde{Y}_{3}\right) \sim 1$.

\subsubsection{Flavour violation in $U(3)^{3}$ models}

The leading contributions to the flavour-changing dipole operators in Eq. (25) vanish in models with $U(3)^{3}$ flavour symmetry and left- or right-handed compositeness. Subleading contributions to the unprimed Wilson coefficients arise, as discussed in Sect. 4.2. The strongest bound is on the coefficient $C_{b s V}$, for which a crude estimate yields

$\delta C_{b s V} \sim V_{t s} \frac{Y^{2}}{m_{Q}^{2}} \frac{s_{R t}}{s_{L t} Y}$,

leading to the bound

$\frac{Y^{2}}{m_{Q}^{2}} \frac{s_{R t}}{s_{L t} Y} \lesssim\left(\frac{1}{0.6 \mathrm{TeV}}\right)^{2}$ 
Table 7 Bound on the quantity $\left(\frac{Y \tilde{Y}}{m_{Q} m_{R}}-\frac{Y_{3} \tilde{Y}_{3}}{m_{Q 3} m_{R 3}}\right)^{-1 / 2}$ in $U(2)^{3}$ flavour models with left-handed compositeness

\begin{tabular}{llll}
\hline Operator & Doublet $(\mathrm{TeV})$ & Triplet $(\mathrm{TeV})$ & Bidoublet $(\mathrm{TeV})$ \\
\hline$Q_{b s V}$ & 0.37 & 0.52 & 0.22 \\
\hline
\end{tabular}

\subsubsection{Flavour violation in $U(2)^{3}$ models}

In $U(2)^{3}$ flavour models with left-handed compositeness, the leading contributions to the Wilson coefficients read

$C_{b q V}=V_{t b} V_{t q}^{*}\left[a_{d V}\left(\frac{Y \tilde{Y}}{m_{Q^{m}}}-\frac{Y_{3} \tilde{Y}_{3}}{m_{Q 3} m_{R 3}}\right)\right]$ for $q=d, s$,

and all other coefficients are negligible. Again, there are no additional $O(1)$ factors. Since the coefficients relevant for $b \rightarrow d$ and $b \rightarrow s$ transitions are correlated in these models, it is sufficient to quote the (stronger) bound derived from the $B \rightarrow X_{s} \gamma$ branching ratio. It is shown in Table 7 .

In $U(2)^{3}$ models with right-handed compositeness, the Wilson coefficients vanish at leading order in the compositeelementary mixings. Beyond the leading order, there are contributions both in left- and right-handed compositeness analogous to the ones in $U(3)^{3}$ models. They give rise to a bound similar to Eq. (50).

\section{Comparison with the literature}

Since some of the dipole operators have been considered in the literature in various models similar to the ones we studied here, we present below a detailed comparison of our findings with those of existing analyses. We find mostly agreement, but also some important differences.

- In Ref. [23], the $B \rightarrow X_{s} \gamma$ branching ratio has been calculated in a model similar to our doublet model, the difference being that the right-handed quarks do not mix with an $S U(2)_{R}$ doublet, but with two singlets, such that the strong Yukawa couplings explicitly break custodial symmetry. For the leading-order contribution to the Wilson coefficient from $W, Z$ or Higgs loops, this difference is, however, irrelevant. Up to an overall sign, we agree with the result for the charged Goldstone $(W)$ contribution $\left[\left(a_{d \gamma}\right)_{W}=5 / 12\right.$ in our language $]$, but disagree with the result for the neutral contribution [we find $\left(a_{d \gamma}\right)_{h}=-1 / 8$ and $\left.\left(a_{d \gamma}\right)_{Z}=-1 / 24\right]$.

- In Ref. [24], the $B \rightarrow X_{s} \gamma$ branching ratio and the observable $\epsilon^{\prime} / \epsilon$ have been estimated in a Randall-Sundrum framework. In the anarchic doublet model, which most closely resembles their setup, the bounds we obtain from these processes are consistent with the ones found in that reference.

- Ref. [34] has given an estimate of the leading contribution to the $B \rightarrow X_{s} \gamma$ branching ratio from loops with a Higgs boson or a charged or neutral Goldstone boson ( $W$ or $Z$ ), corresponding to our Eq. (25), in the anarchic bidoublet model. We disagree with the fact that the leading-order contribution does not involve the wrong-chirality Yukawa couplings. In the same reference, EDMs in $U(3)^{3}$ models with left-handed compositeness were discussed, and it was claimed that the new CP-violating phase does not enter the EDM at leading order, since it can be shifted to $\tilde{Y}$. Our analysis shows that the converse is true: the leading contribution to the EDM is proportional to $\tilde{Y}$. The bounds we obtain are shown in Table 6.

- The authors of Ref. [28] have performed a 5D calculation of $b \rightarrow q \gamma$ processes in a Randall-Sundrum setup. The choice of fermion representations is similar to our triplet model, but the right-handed up-type quarks couple to a singlet. Furthermore, the model effectively has $\tilde{Y}=Y$. Our numerical estimates for the bound from the $B \rightarrow X_{s, d} \gamma$ branching fractions are compatible with the numerical analysis presented in that work.

- Ref. [26] contains a thorough analysis of the $B \rightarrow X_{s} \gamma$ branching ratio and the observable $\epsilon^{\prime} / \epsilon$, closely following [23], in the triplet and bidoublet models (denoted TS5 and TS10, respectively) with flavour anarchy, setting $\tilde{Y}=Y$. While we agree on the overall form of the results, we have several differences in the coefficients $a_{q V}$. We present the details of our calculation in the bidoublet model in Appendix 10.

- In Ref. [25], the $c \rightarrow u g$ dipole transition was considered in Randall-Sundrum models in the context of $\Delta A_{\mathrm{CP}}$ in $D \rightarrow K K, \pi \pi$ decays. In particular, the authors emphasise the dependence of the leading contribution on the wrong-chirality Yukawa coupling, and we confirm their findings in our 4D setup.

\section{Conclusions}

Dipole operators with quarks and an on-shell photon or gluon are generated at the one-loop level in theories based on the mechanism of partial compositeness, where the quarks get their masses by mixing with heavy vector-like "composite" fermions. Paradigm examples in this class of models are composite Higgs models or warped extra dimensions. The dipole operators contribute to numerous observables, like EDMs or FCNC decays, which can then be used to constrain these models. In this paper, we have performed an analysis of all dipole operators in the quark sector that are constrained by experiment within a simple 4D setup with a single set of vector-like fermions. We have chosen this simple framework 
so as to be able to study the effects of choosing different representations for the composite fermion fields and of imposing different flavour structures in the strong sector. Our main findings can be summarised as follows:

- The leading contributions to the Wilson coefficients, discussed in Sect. 4.1, typically come from diagrams with a heavy fermion and a $W, Z$ or Higgs in the loop. These contributions are proportional to the "wrong-chirality" Yukawa couplings $\tilde{Y}$.

- Beyond these leading contributions there exist a number of subleading effects, which we have categorised systematically and discussed qualitatively in Sect. 4.2. They can be relevant, e.g., in models where the wrong-chirality Yukawas are absent or suppressed. In the case of $b \rightarrow s, d$ transitions, these subleading contributions can be comparable to the leading ones (due to the sizable degrees of compositeness of the top quark), while in all other cases they are typically suppressed by at least an order of magnitude for $\mathrm{TeV}$-scale resonances.

- In models with anarchic flavour and CP structures, the neutron EDM leads to a stringent constraint. If the average Yukawa couplings $Y$ and wrong-chirality Yukawa couplings $\tilde{Y}$ are of $O(1)$, this implies that the average fermion resonance mass scale should be above $4 \mathrm{TeV}$ or so. For larger Yukawa couplings, the bounds become even stronger. Apart from the neutron EDM bound, there is a multitude of bounds in the $1-2 \mathrm{TeV}$ ballpark, as summarised in Table 5.

- In models in which the wrong-chirality Yukawa couplings are absent, the bounds from dipole operators are mild, even for an anarchic flavour and CP structures.

- In models featuring a $U(3)^{3}$ flavour symmetry broken only by left- or right-handed composite-elementary mixings, there is a bound from the neutron EDM that is as strong as in the anarchic case. It can be avoided by assuming the wrong-chirality Yukawa coupling to be real (or absent).

- In models with a $U(2)^{3}$ flavour symmetry broken only by left- or right-handed composite-elementary mixings, the EDM bound can be avoided alternatively by raising the mass of the composite fermions of the first two generations.

- In $U(2)^{3}$ flavour models with left-handed compositeness, there are bounds from flavour-violating dipoles, which are, however, very mild.

While our results have been obtained in the simple framework defined in Sect. 2, in more complete models the details of the analysis can be different. Here we only briefly comment on models where the Higgs is a pseudo Nambu-Goldstone boson (PNGB) [4,5], which are particularly well motivated in view of the lightness of the Higgs boson. Strictly speaking, these models are not a special case of the Lagrangian defined in Sect. 2. Given that the dominant contributions to the dipole operators come from diagrams with a heavy fermion and a Higgs, $W$ or $Z$ and does not depend on the details of the composite spin-1 sector, it is instructive to compare the fermion mass matrices in the two cases. For example, in the minimal composite Higgs model referred to as MCHM5 [57], the fermion mass matrix can be written in a form (see e.g. [58]) that, to leading order in the expansion in the Higgs vacuum expectation value, corresponds to the mass matrix in our bidoublet model (see Appendix 8) with $\tilde{Y}=Y$. Thus we expect that with the identification $\tilde{Y}=Y$ our results for the leading contributions to the Wilson coefficients also hold in composite PNGB models, up to $O(1)$ factors.

There are several ways to extend our analysis. Also in the charged lepton sector, dipole operators arise and contribute to $\ell_{i} \rightarrow \ell_{j} \gamma$ decays, to the electron EDM or to the muon anomalous magnetic moment [53,59,60] (see also [61-63]). Finally, a global numerical analysis of all contributions to $\Delta F=1$ and $\Delta F=2$ processes, taking into account electroweak constraints, would be interesting. We leave this to a future publication.

Acknowledgments We thank Dario Buttazzo, Michele Redi, Filippo Sala, Andrea Tesi, and Natascia Vignaroli for useful discussions. This research has been supported by the Advanced Grant EFT4LHC of the European Research Council (ERC), the Cluster of Excellence Precision Physics, Fundamental Interactions and Structure of Matter (PRISMA EXC 1098), and grant 05H12UME of the German Federal Ministry for Education and Research (BMBF). The research of D.S. is supported by the DFG cluster of excellence "Origin and Structure of the Universe".

Open Access This article is distributed under the terms of the Creative Commons Attribution License which permits any use, distribution, and reproduction in any medium, provided the original author(s) and the source are credited.

Funded by $\mathrm{SCOAP}^{3}$ / License Version CC BY 4.0.

\section{Appendix A: Fermion mass matrices}

In this section we list the mass matrices of the heavy fermion resonances in all three models. In the doublet model, they are given by

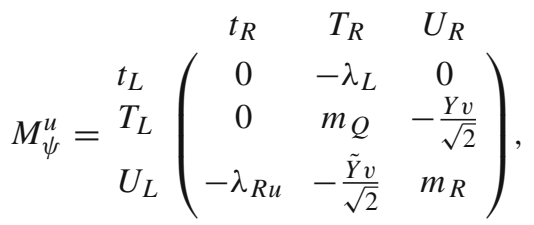

$$
\begin{aligned}
& M_{\psi}^{d}=\begin{array}{c}
b_{L} \\
B_{L} \\
D_{L}
\end{array}\left(\begin{array}{ccc}
b_{R} & B_{R} & D_{R} \\
0 & -\lambda_{L} & 0 \\
0 & m_{Q} & -\frac{Y v}{\sqrt{2}} \\
-\lambda_{R d} & -\frac{\tilde{Y} v}{\sqrt{2}} & m_{R}
\end{array}\right) .
\end{aligned}
$$


In the triplet model, they are

$$
\begin{aligned}
& \begin{array}{c}
t_{L} \\
U_{L}^{u}=U_{\psi}^{\prime} \\
T_{L} \\
T_{2 / 3 L}
\end{array}\left(\begin{array}{ccccc}
0 & U_{R} & U_{R}^{\prime} & T_{R} & T_{2 / 3 R} \\
-\lambda_{R u} & m_{R} & 0 & -\frac{\tilde{Y} v}{2} & \frac{\tilde{Y} v}{2} \\
0 & 0 & m_{R} & -\frac{\tilde{Y} v}{2} & \frac{\tilde{Y} v}{2} \\
0 & -\frac{Y v}{2} & -\frac{Y v}{2} & m_{Q} & 0 \\
0 & \frac{Y v}{2} & \frac{Y v}{2} & 0 & m_{Q}
\end{array}\right), \\
& M_{\psi}^{d}=\begin{array}{c}
b_{L} \\
D_{L} \\
D_{L}^{\prime} \\
B_{L}
\end{array}\left(\begin{array}{cccr}
0 & 0 & 0 & -\lambda_{L} \\
-\lambda_{R d} & m_{R} & 0 & -\frac{\tilde{Y} v}{\sqrt{2}} \\
0 & 0 & m_{R} & -\frac{\tilde{Y} v}{\sqrt{2}} \\
0 & -\frac{Y v}{\sqrt{2}} & -\frac{Y v}{\sqrt{2}} & m_{Q}
\end{array}\right),
\end{aligned}
$$

and

$$
M_{\psi}^{5 / 3}=T_{5 / 3 L} U_{5 / 3 L}\left(\begin{array}{ccc}
T_{5 / 3 R} & U_{5 / 3 R} & U_{5 / 3 R}^{\prime} \\
m_{Q} & -\frac{Y v}{\sqrt{2}} & -\frac{Y v}{\sqrt{2}} \\
-\frac{\tilde{Y} v}{\sqrt{2}} & m_{R} & 0 \\
-\frac{\tilde{Y} v}{\sqrt{2}} & 0 & m_{R}
\end{array}\right) .
$$

In the bidoublet model, they are

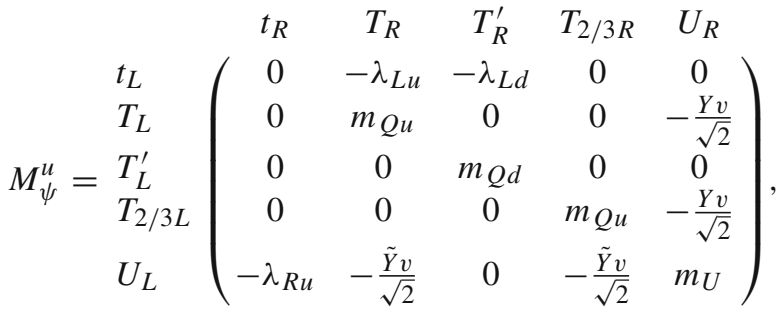

$$
\begin{aligned}
& b_{R} \quad B_{R} \quad B_{R}^{\prime} \quad B_{-1 / 3 R} \quad D_{R} \\
& M_{\psi}^{d}=\begin{array}{l}
b_{L} \\
B_{L} \\
B_{L}^{\prime} \\
B_{-1 / 3 L} \\
D_{L}
\end{array}\left(\begin{array}{ccccc}
0 & -\lambda_{L u} & -\lambda_{L d} & 0 & 0 \\
0 & m_{Q u} & 0 & 0 & 0 \\
0 & 0 & m_{Q d} & 0 & -\frac{Y v}{\sqrt{2}} \\
0 & 0 & 0 & m_{Q u} & -\frac{Y v}{\sqrt{2}} \\
-\lambda_{R d} & 0 & -\frac{\tilde{Y} v}{\sqrt{2}} & -\frac{\tilde{Y} v}{\sqrt{2}} & m_{D}
\end{array}\right)
\end{aligned}
$$

In the discussion of our results we have switched from the mixing parameters $\lambda_{i}$ to the sines of the mixing angles determining the degree of compositeness. The compositeelementary mixings are in general given by $s_{i} \equiv \sin \varphi_{i} \approx$ $\tan \varphi_{i}=\lambda_{i} / m_{i}$; more specifically, for the doublet and triplet models

$$
\begin{aligned}
& s_{L t}=s_{L b} \equiv s_{L}=\frac{\lambda_{L}}{\sqrt{m_{Q}^{2}+\lambda_{L}^{2}}}, \\
& s_{R t}=\frac{\lambda_{R u}}{\sqrt{m_{R}^{2}+\lambda_{R u}^{2}}}, \quad s_{R b}=\frac{\lambda_{R d}}{\sqrt{m_{R}^{2}+\lambda_{R d}^{2}}},
\end{aligned}
$$

whereas in the bidoublet model

$$
\begin{aligned}
& s_{L t}=\frac{\lambda_{L u}}{\sqrt{m_{Q u}^{2}+\lambda_{L u}^{2}}}, \quad s_{R t}=\frac{\lambda_{R u}}{\sqrt{m_{U}^{2}+\lambda_{R u}^{2}}}, \\
& s_{L b}=\frac{\lambda_{L d}}{\sqrt{m_{Q d}^{2}+\lambda_{L d}^{2}}}, \quad s_{R b}=\frac{\lambda_{R d}}{\sqrt{m_{D}^{2}+\lambda_{R d}^{2}}} .
\end{aligned}
$$

\section{Appendix B: Model-independent formulae for the Wilson coefficients}

Here we give the exact analytical expressions for the oneloop Wilson coefficients, which were used to obtain the approximate expressions given in the text. The Wilson coefficients of the $q_{i} \rightarrow q_{j} \gamma$ dipole operators, as defined in Eqs. (10)-(12) with $i>j$, can be written as

$$
\begin{aligned}
\mathcal{C}_{q_{i} q_{j} \gamma, g}= & \sum_{\psi, X} \frac{1}{m_{q_{i}} m_{X}^{2}}\left(m_{q_{i}} V_{i \psi X}^{L *} V_{j \psi X}^{L}+m_{q_{j}} V_{i \psi X}^{R *} V_{j \psi X}^{R}\right) \\
& \times F_{X}^{1}\left(Q_{\psi}, Q_{X}, x\right) \\
& +\frac{1}{m_{q_{i}} m_{X}^{2}}\left(m_{\psi} V_{i \psi X}^{L *} V_{j \psi X}^{R}\right) F_{X}^{2}\left(Q_{\psi}, Q_{X}, x\right),
\end{aligned}
$$

where $\psi$ denotes the fermion entering the loop and $X$ can be either vector, scalar or a heavy gluon resonance. The parameter $x$ is given by $x=m_{\psi}^{2} / m_{X}^{2}$. The expression for the primed Wilson coefficient $\mathcal{C}_{q_{i} q_{j}, g}^{\prime}$ can be obtained from Eq. (59) by interchanging $L \leftrightarrow R$. The loop functions are defined as

$$
\begin{aligned}
& F_{V}^{1}\left(Q_{\psi}, Q_{V}, x\right) \\
& =Q_{\psi} \frac{\left(5 x^{4}-14 x^{3}+39 x^{2}-18 x^{2} \log x-38 x+8\right)}{24(x-1)^{4}} \\
& \quad+Q_{V} \frac{\left(4 x^{4}-49 x^{3}+18 x^{3} \log x+78 x^{2}-43 x+10\right)}{24(x-1)^{4}}, \\
& F_{V}^{2}\left(Q_{\psi}, Q_{V}, x\right)=Q_{\psi} \frac{\left(-x^{3}-3 x+6 x \log x+4\right)}{4(x-1)^{3}} \\
& \quad+Q_{V} \frac{\left(-x^{3}+12 x^{2}-6 x^{2} \log x-15 x+4\right)}{4(x-1)^{3}}, \\
& F_{S}^{1}\left(Q_{\psi}, Q_{S}, x\right)=Q_{\psi} \frac{\left(-x^{3}+6 x^{2}-3 x-6 x \log x-2\right)}{24(x-1)^{4}} \\
& \quad+Q_{S} \frac{\left(2 x^{3}+3 x^{2}-6 x^{2} \log x-6 x+1\right)}{24(x-1)^{4}}
\end{aligned}
$$


Table 8 Charge parameters for the loop functions (63) depending on contribution

\begin{tabular}{llllllll}
\hline $\mathcal{C}$ & $X$ & $Q_{F}$ & $Q_{X}$ & $\mathcal{C}$ & $X$ & $Q_{F}$ & $Q_{X}$ \\
\hline $\mathcal{C}_{d d \gamma}$ & $h, Z, \rho^{0}$ & $-1 / 3$ & 0 & $\mathcal{C}_{\text {uи }}$ & $h, Z, \rho^{0}$ & $2 / 3$ & 0 \\
& $W^{+}, \rho^{+}$ & $-4 / 3$ & 1 & & $W^{+}, \rho^{+}$ & $-1 / 3$ & 1 \\
& $W^{-}, \rho^{-}$ & $2 / 3$ & -1 & & $W^{-}, \rho^{-}$ & $5 / 3$ & -1 \\
& $G^{*}$ & $-4 / 9$ & 0 & & $G^{*}$ & $8 / 9$ & 0 \\
$\mathcal{C}_{d d g}$ & $h, W, \rho$ & 1 & 0 & $\mathcal{C}_{\text {uug }}$ & $h, W, \rho$ & 1 & 0 \\
& $G^{*}$ & $-1 / 6$ & $3 / 2$ & & $G^{*}$ & $-1 / 6$ & $3 / 2$ \\
\hline
\end{tabular}

$$
\begin{aligned}
& F_{S}^{2}\left(Q_{\psi}, Q_{S}, x\right)=Q_{\psi} \frac{\left(-x^{2}+4 x-2 \log x-3\right)}{4(x-1)^{3}} \\
& \quad+Q_{S} \frac{\left(x^{2}-2 x \log x-1\right)}{4(x-1)^{3}} .
\end{aligned}
$$

Note that the charge parameters $Q_{\psi, V, S}$ in the loop functions are not necessarily the electric charges of the corresponding particles but can be colour factors in the cases of either the external gauge field being a gluon or the loop involving heavy gluon resonances. A complete reference is given in Table 8 . The couplings $V_{i \psi X}^{L / R}$ in Eq. (59) are defined as follows:

$$
\sim_{V}^{\mu}=i \bar{q}_{i} \gamma^{\mu}\left(V_{i \psi V}^{L} P_{L}+V_{i \psi V}^{R} P_{R}\right) V_{\mu} \psi
$$

\section{Appendix C: Calculation of leading contribution in the bidoublet model}

Here we illustrate the calculation for the leading-order correction to $\mathcal{C}_{q q \gamma}$ in the bidoublet model for one generation.
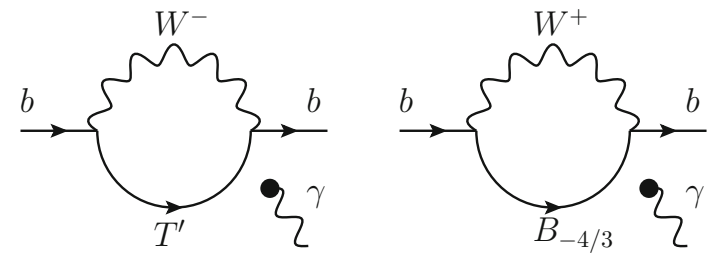

Fig. 2 Diagrams contributing to the leading correction in the bidoublet model. The blob on the photon leg denotes the photon either coupling to the loop fermion or the $W$ boson. We use the same names for the fermion
This contribution is governed by diagrams with a heavy fermion and a $W, Z$ or Higgs in the loop, as shown in Fig. 2. We obtain

$\mathcal{C}_{b b \gamma}=\sum_{\psi, X} \frac{m_{\psi}}{m_{b} m_{X}^{2}} V_{b \psi X}^{R} V_{b \psi X}^{L *} F_{X}\left(Q_{\psi}, Q_{X}, x\right)$,

where $m_{\psi}$ and $m_{X}$ are the masses of the fermion and the boson in the loop. The $V_{b \psi X}^{L, R}$ are the fermion-gauge couplings in the mass eigenbasis, as defined in Appendix 9. For the loop functions, we use the approximations

$$
F_{V}\left(Q_{\psi}, Q_{V}, x\right) \approx-\frac{Q_{\psi}+Q_{V}}{4}, \quad F_{S}\left(Q_{\psi}, x\right) \approx-\frac{Q_{\psi}}{x},
$$

where we only kept the first non-vanishing order for $x \rightarrow \infty$. The gauge couplings up to quadratic order of the compositethe mass eigenstates to order $O(v)$. We have followed the convention of setting $m_{Q u}=m_{Q d} \rightarrow m_{Q}$ and $m_{U}=$ $m_{D} \rightarrow m_{R}$ everywhere. Putting all the pieces together, we find the contributions listed in Table 11. Summing up these contributions, we have obtained the result from Sect. 4, i.e.

$\mathcal{C}_{b b \gamma}=\sum_{i} c_{i}=a_{d \gamma} \frac{Y \tilde{Y}}{m_{Q} m_{R}}$

with $a_{d \gamma}=-1 / 2$.

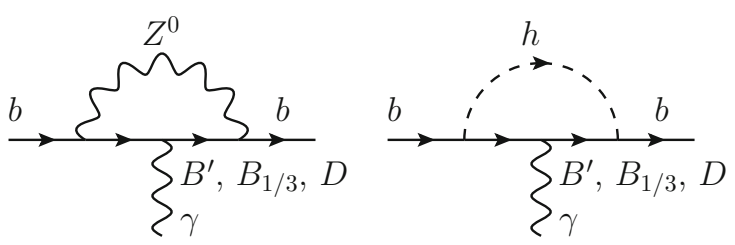

mass eigenstates as for the fermion fields in the composite-elementary basis. The mass eigenstates are understood to correspond to the fields in the original basis with whom they have the largest admixtures elementary mixings are given in Table 9. Table 10 lists 
Table 9 Couplings of the $b$ and the loop fermion to the loop boson in the bidoublet model

\begin{tabular}{|c|c|c|c|}
\hline$\psi$ & $X$ & $V_{b \psi X}^{R}$ & $V_{b \psi X}^{L}$ \\
\hline \multirow{3}{*}{$T^{\prime}$} & \multirow{2}{*}{$W_{\mu}^{-}$} & $g Y \tilde{Y} v^{2} s_{L b}$ & $g Y v s_{R b}$ \\
\hline & & $\overline{2 \sqrt{2} m_{R} m_{Q}}$ & $-\frac{2 m_{Q}}{}$ \\
\hline & & $g Y \tilde{Y} v^{2} s_{L b}$ & $-\frac{g Y v s_{R b}}{}$ \\
\hline$B_{-4 / 3}$ & $W_{\mu}^{+}$ & $\overline{2 \sqrt{2} m_{Q} m_{R}}$ & $2 m_{Q}$ \\
\hline \multirow{2}{*}{$B^{\prime}$} & \multirow{2}{*}{$Z_{\mu}^{0}$} & $g Y v s_{R b}$ & $g Y\left(Y m_{R}+\tilde{Y} m_{Q}\right) v^{2} s_{L b}$ \\
\hline & & $2 \sqrt{2} m_{Q \sqrt{1-s_{W}^{2}}}$ & $4 m_{R}\left(m_{R}^{2}-m_{Q}^{2}\right) \sqrt{1-s_{W}^{2}}$ \\
\hline \multirow{2}{*}{$B_{1 / 3}$} & \multirow{2}{*}{$Z_{\mu}^{0}$} & $g Y v s_{R b}$ & $g Y\left(Y m_{Q} m_{R}-\tilde{Y} m_{Q}^{2}+2 \tilde{Y} m_{R}^{2}\right) v^{2} s_{L b}$ \\
\hline & & $2 \sqrt{2} m_{Q \sqrt{1-s_{W}^{2}}}$ & $4 m_{Q} m_{R}\left(m_{R}^{2}-m_{Q}^{2}\right) \sqrt{1-s_{W}^{2}}$ \\
\hline$D$ & $Z_{\mu}^{0}$ & 0 & $-\frac{g Y v s_{L b}}{2 \sqrt{2} m_{R} \sqrt{1-s_{W}^{2}}}$ \\
\hline \multirow{2}{*}{$B^{\prime}$} & \multirow{2}{*}{$h$} & $Y s_{R b}$ & $Y v\left(\tilde{Y} m_{Q}^{2}-2 \tilde{Y} m_{R}^{2}-Y m_{Q} m_{R}\right) s_{L b}$ \\
\hline & & $\sqrt{2}$ & $2 m_{R}\left(m_{R}^{2}-m_{Q}^{2}\right)$ \\
\hline \multirow{2}{*}{$B_{1 / 3}$} & \multirow{2}{*}{$h$} & $\underline{Y s_{R b}}$ & $Y v\left(\tilde{Y} m_{Q}^{2}-2 \tilde{Y} m_{R}^{2}-Y m_{Q} m_{R}\right) s_{L b}$ \\
\hline & & $\sqrt{2}$ & $2 m_{R}\left(m_{R}^{2}-m_{Q}^{2}\right)$ \\
\hline \multirow{2}{*}{$D$} & \multirow{2}{*}{$h$} & $Y \tilde{Y} v\left(m_{R}^{2}-2 m_{Q}^{2}\right) s_{R b} \quad Y^{2} v s_{R b}$ & $Y s_{L b}$ \\
\hline & & $m L\left(m_{Q}^{2}-m_{R}^{2}\right)-\overline{m_{Q}^{2}-m_{R}^{2}}$ & $\overline{\sqrt{2}}$ \\
\hline
\end{tabular}

Table 10 Mass eigenstates of the involved fermions at order $O(v)$ in the bidoublet model

\begin{tabular}{llllll}
\hline$b$ & $B^{\prime}$ & $B_{1 / 3}$ & $D$ & $T^{\prime}$ & $B_{-4 / 3}$ \\
\hline$\frac{Y v}{\sqrt{2}} s_{L b} s_{R b}$ & $m_{Q}$ & $m_{Q}$ & $m_{R}+\frac{m_{R}}{2} s_{R b}^{2}$ & $m_{Q}$ & $m_{Q}$ \\
\hline
\end{tabular}

Table 11 Contributions relevant to the leading order correction of $C_{b b \gamma}$ in the bidoublet model

\begin{tabular}{lll}
\hline Loop Fermion & Loop Boson & $c_{i}$ \\
\hline$T^{\prime}$ & $W_{\mu}^{-}$ & $\frac{5 Y \tilde{Y}}{12 m_{Q} m_{R}}$ \\
$B_{-4 / 3}$ & $W_{\mu}^{+}$ & $-\frac{7 Y Y}{12 m_{Q} \tilde{m}_{R}}$ \\
$B^{\prime}$ & $Z_{\mu}^{0}$ & $\frac{Y{ }_{Y} m_{Q}}{24\left(m_{R}^{3}-m_{Q}^{2} m_{R}\right)}$ \\
$B_{1 / 3}$ & $Z_{\mu}^{0}$ & $\frac{Y \tilde{Y}\left(m_{Q}^{2}-2 m_{R}^{2}\right)}{24\left(m_{Q} m_{R}^{3}-m_{Q}^{3} m_{R}\right)}$ \\
$D$ & $Z_{\mu}^{0}$ & 0 \\
$B^{\prime}$ & Higgs & $\frac{Y \tilde{Y}\left(m_{Q}^{2}-2 m_{R}^{2}\right)}{24\left(m_{Q} m_{R}^{3}-m_{Q}^{3} m_{R}\right)}$ \\
& & $\frac{Y \tilde{Y}\left(m_{Q}^{2}-2 m_{R}^{2}\right)}{24\left(m_{Q} m_{R}^{3}-m_{Q}^{3} m_{R}\right)}$ \\
$B_{1 / 3}$ & Higgs & $\frac{Y \tilde{Y}\left(2 m_{Q}^{2}-m_{R}^{2}\right)}{12\left(m_{Q}^{3} m_{R}-m_{Q} m_{R}^{3}\right)}$ \\
& & Higgs
\end{tabular}

\section{References}

1. ATLAS Collaboration, G. Aad et al., Observation of a new particle in the search for the Standard Model Higgs boson with the ATLAS detector at the LHC, Phys. Lett. B716 (2012) 1-29. arXiv: 1207.7214

2. C.M.S. Collaboration, S. Chatrchyan et al., Observation of a new boson at a mass of $125 \mathrm{GeV}$ with the CMS experiment at the LHC, Phys. Lett. B716, 30-61 (2012). arXiv:1207.7235

3. L. Randall, R. Sundrum, A large mass hierarchy from a small extra dimension. Phys. Rev. Lett. 83, 3370-3373 (1999). hep-ph/9905221

4. R. Contino, Y. Nomura, A. Pomarol, Higgs as a holographic pseudo-Goldstone boson. Nucl. Phys. B671, 148-174 (2003). hep-ph/0306259

5. K. Agashe, R. Contino, A. Pomarol, The minimal composite Higgs model. Nucl. Phys. B719, 165-187 (2005). hep-ph/0412089

6. R. Contino, T. Kramer, M. Son, R. Sundrum, Warped/composite phenomenology simplified. JHEP 0705, 074 (2007). hep-ph/0612180

7. R. Barbieri, B. Bellazzini, V.S. Rychkov, A. Varagnolo, The Higgs boson from an extended symmetry. Phys. Rev. D76, 115008 (2007). arXiv:0706.0432

8. S. De Curtis, M. Redi, A. Tesi, The 4D composite Higgs. JHEP 1204, 042 (2012). arXiv:1110.1613

9. G. Panico, A. Wulzer, The discrete composite Higgs model. JHEP 1109, 135 (2011). arXiv:1106.2719

10. D. Marzocca, M. Serone, J. Shu, General composite Higgs models. JHEP 1208, 013 (2012). arXiv: 1205.0770

11. B. Bellazzini, C. Csáki, J. Serra, Composite Higgses. arXiv: 1401.2457

12. D.B. Kaplan, Flavor at SSC energies: a new mechanism for dynamically generated fermion masses. Nucl. Phys. B365, 259-278 (1991) 
13. Y. Grossman, M. Neubert, Neutrino masses and mixings in nonfactorizable geometry. Phys. Lett. B474, 361-371 (2000). hep-ph/9912408

14. S.J. Huber, Q. Shafi, Fermion masses, mixings and proton decay in a Randall-Sundrum model. Phys. Lett. B498, 256-262 (2001). hep-ph/0010195

15. T. Gherghetta, A. Pomarol, Bulk fields and supersymmetry in a slice of AdS. Nucl. Phys. B586, 141-162 (2000). hep-ph/0003129

16. K. Agashe, G. Perez, A. Soni, Flavor structure of warped extra dimension models. Phys. Rev. D71, 016002 (2005). hep-ph/0408134

17. C. Csaki, A. Falkowski, A. Weiler, The flavor of the composite pseudo-Goldstone Higgs. JHEP 0809, 008 (2008). arXiv:0804.1954

18. M. Blanke, A.J. Buras, B. Duling, S. Gori, A. Weiler, $\Delta \mathrm{F}=2$ observables and fine-tuning in a warped extra dimension with custodial protection. JHEP 0903, 001 (2009). arXiv:0809.1073

19. M. Bauer, S. Casagrande, U. Haisch, M. Neubert, Flavor physics in the Randall-Sundrum model: II. Tree-level weak-interaction processes. JHEP 1009, 017 (2010). arXiv:0912.1625

20. K. Agashe, A. Delgado, M.J. May, R. Sundrum, RS1, custodial isospin and precision tests. JHEP 0308, 050 (2003). hep-ph/0308036

21. K. Agashe, R. Contino, L. Da Rold, A. Pomarol, A custodial symmetry for Z $b \bar{b}$. Phys. Lett. B641, 62-66 (2006). hep-ph/0605341

22. K. Agashe, G. Perez, A. Soni, B-factory signals for a warped extra dimension. Phys. Rev. Lett. 93, 201804 (2004). hep-ph/0406101

23. K. Agashe, A. Azatov, L. Zhu, Flavor violation tests of warped/composite SM in the two-site approach. Phys. Rev. D79, 056006 (2009). arXiv:0810.1016

24. O. Gedalia, G. Isidori, G. Perez, Combining direct and indirect Kaon CP violation to constrain the warped KK scale. Phys. Lett. B682, 200-206 (2009). arXiv:0905.3264

25. C. Delaunay, J.F. Kamenik, G. Perez, L. Randall, Charming CP violation and dipole operators from RS flavor anarchy. JHEP 1301, 027 (2013). arXiv: 1207.0474

26. N. Vignaroli, $\Delta F=1$ constraints on composite Higgs models with LR parity. Phys. Rev. D86, 115011 (2012). arXiv:1204.0478

27. C. Csaki, Y. Grossman, P. Tanedo, Y. Tsai, Warped penguin diagrams. Phys. Rev. D83, 073002 (2011). arXiv:1004.2037

28. M. Blanke, B. Shakya, P. Tanedo, Y. Tsai, The birds and the Bs in RS: The $b \rightarrow s \gamma$ penguin in a warped extra dimension. JHEP 1208, 038 (2012). arXiv: 1203.6650

29. M. Beneke, P. Dey, J. Rohrwild, The muon anomalous magnetic moment in the Randall-Sundrum model. JHEP 1308, 010 (2013). arXiv: 1209.5897

30. R. Barbieri, D. Buttazzo, F. Sala, D.M. Straub, A. Tesi, A $125 \mathrm{GeV}$ composite Higgs boson versus flavour and electroweak precision tests. JHEP 1305, 069 (2013). arXiv:1211.5085

31. D.M. Straub, Anatomy of flavour-changing $Z$ couplings in models with partial compositeness. JHEP 1308, 108 (2013). arXiv: 1302.4651

32. R. Barbieri, G. Isidori, D. Pappadopulo, Composite fermions in electroweak symmetry breaking. JHEP 0902, 029 (2009). arXiv:0811.2888

33. G. Cacciapaglia, C. Csaki, J. Galloway, G. Marandella, J. Terning et al., A GIM mechanism from extra dimensions. JHEP 0804, 006 (2008). arXiv:0709.1714

34. M. Redi, A. Weiler, Flavor and CP invariant composite Higgs models. JHEP 1111, 108 (2011). arXiv:1106.6357

35. R. Barbieri, D. Buttazzo, F. Sala, D.M. Straub, Flavour physics from an approximate $U(2)^{3}$ symmetry. JHEP 1207, 181 (2012). arXiv: 1203.4218

36. G. Buchalla, A.J. Buras, M.E. Lautenbacher, Weak decays beyond leading logarithms. Rev. Mod. Phys. 68, 1125-1144 (1996). hep-ph/9512380
37. A.J. Buras, L. Merlo, E. Stamou, The impact of flavour changing neutral gauge Bosons on $\bar{B} \rightarrow X_{s} \gamma$. JHEP 1108, 124 (2011). arXiv: 1105.5146

38. M. Pospelov, A. Ritz, Neutron EDM from electric and chromoelectric dipole moments of quarks. Phys. Rev. D63, 073015 (2001). hep-ph/0010037

39. C. Baker, D. Doyle, P. Geltenbort, K. Green, M. van der Grinten, et al., An improved experimental limit on the electric dipole moment of the neutron, Phys. Rev. Lett. 97 (2006) 131801. hep-ex/0602020

40. J. Hewett, H. Weerts, R. Brock, J. Butler, B. Casey, et al., Fundamental physics at the intensity frontier. arXiv:1205.2671

41. E. Braaten, C.-S. Li, T.-C. Yuan, The evolution of Weinberg's gluonic CP violation operator. Phys. Rev. Lett. 64, 1709 (1990)

42. D. Chang, W.-Y. Keung, C. Li, T. Yuan, QCD corrections to CP violation from color electric dipole moment of $b$ quark. Phys. Lett. B241, 589 (1990)

43. J.F. Kamenik, M. Papucci, A. Weiler, Constraining the dipole moments of the top quark. Phys. Rev. D85, 071501 (2012). arXiv: 1107.3143

44. F. Sala, A bound on the charm chromo-EDM and its implications. arXiv: 1312.2589

45. Heavy Flavor Averaging Group Collaboration, Y. Amhis et al., Averages of $b$ hadron, $c$ hadron, and $\tau$ lepton properties as of early 2012. arXiv: 1207.1158

46. M. Misiak, H. Asatrian, K. Bieri, M. Czakon, A. Czarnecki et al., Estimate of $B\left(\bar{B} \rightarrow X_{s} \gamma\right)$ at $O\left(\alpha_{s}^{2}\right)$. Phys. Rev. Lett. 98, 022002 (2007). hep-ph/0609232

47. T. Becher, M. Neubert, Analysis of $\operatorname{Br}\left(\bar{B} \rightarrow X_{s} \gamma\right)$ at NNLO with a cut on photon energy. Phys. Rev. Lett. 98, 022003 (2007). hep-ph/0610067

48. A. Crivellin, L. Mercolli, $B \rightarrow X_{d} \gamma$ and constraints on new physics. Phys. Rev. D84, 114005 (2011). arXiv:1106.5499

49. A.L. Kagan, M. Neubert, QCD anatomy of $\bar{B} \rightarrow X_{s} \gamma$ decays. Eur. Phys. J. C7, 5-27 (1999). hep-ph/9805303

50. P. Mertens, C. Smith, The $s \rightarrow d \gamma$ decay in and beyond the standard model. JHEP 1108, 069 (2011). arXiv: 1103.5992

51. B. Keren-Zur, P. Lodone, M. Nardecchia, D. Pappadopulo, R. Rattazzi, et al., On partial compositeness and the CP asymmetry in charm decays, Nucl. Phys. B867, 429-447 (2013). arXiv: 1205.5803

52. G. Isidori, J.F. Kamenik, Z. Ligeti, G. Perez, Implications of the LHCb evidence for charm CP violation, Phys. Lett. B711, 46-51 (2012). arXiv:1111.4987

53. K. Agashe, A.E. Blechman, F. Petriello, Probing the RandallSundrum geometric origin of flavor with lepton flavor violation. Phys. Rev. D74, 053011 (2006). hep-ph/0606021

54. S. Casagrande, F. Goertz, U. Haisch, M. Neubert, T. Pfoh, Flavor physics in the Randall-Sundrum model: I. Theoretical setup and electroweak precision tests. JHEP 0810, 094 (2008). arXiv:0807.4937

55. G. Giudice, A. Romanino, Electric dipole moments in split supersymmetry. Phys. Lett. B634, 307-314 (2006). hep-ph/0510197

56. D. Becirevic, E. Kou, A. Le Yaouanc, A. Tayduganov, Future prospects for the determination of the Wilson coefficient $C_{7 \gamma}^{\prime}$. JHEP 1208, 090 (2012). arXiv: 1206.1502

57. R. Contino, L. Da Rold, A. Pomarol, Light custodians in natural composite Higgs models. Phys. Rev. D75, 055014 (2007). hep-ph/0612048

58. C. Delaunay, C. Grojean, G. Perez, Modified Higgs physics from composite light flavors. JHEP 1309, 090 (2013). arXiv:1303.5701

59. K. Agashe, Relaxing constraints from lepton flavor violation in 5D flavorful theories. Phys. Rev. D80, 115020 (2009). arXiv:0902.2400

60. M. Redi, Leptons in composite MFV. JHEP 1309, 060 (2013). arXiv: 1306.1525 
61. K. Kannike, M. Raidal, D.M. Straub, A. Strumia, Anthropic solution to the magnetic muon anomaly: the charged see-saw. JHEP 1202, 106 (2012). arXiv:1111.2551

62. R. Dermisek, A. Raval, Explanation of the Muon g-2 anomaly with vector like leptons and its implications for Higgs decays. Phys. Rev. D88, 013017 (2013). arXiv:1305.3522
63. A. Falkowski, D.M. Straub, A. Vicente, Vector-like leptons: Higgs decays and collider phenomenology. JHEP 1405, 092 (2014). arXiv: 1312.5329 